\title{
Barium Isotope Abundances in Meteorites and Their Implications for Early Solar System Evolution
}

K.R. Bermingham ${ }^{\mathrm{a}, \mathrm{b}}$, K. Mezger ${ }^{\mathrm{a}, \mathrm{c}}$, E.E. Scherer ${ }^{\mathrm{a}}$, M. $\operatorname{Horan}^{\mathrm{d}}$, R. Carlson ${ }^{\mathrm{d}}$, D. Upadhyay ${ }^{\mathrm{a}, \mathrm{e}}$, T. Magna, ${ }^{\mathrm{a}, \mathrm{f}}$, A. Pack ${ }^{\mathrm{g}}$

${ }^{a}$ Institut für Mineralogie, Westfälische Wilhelms-Universität, Corrensstraße 24, 48149 Münster, Germany

${ }^{b}$ Isotope Geochemistry Laboratory, Department of Geology, University of Maryland, College Park, MD-20740 USA

${ }^{c}$ Institut für Geologie, Universität Bern, Baltzerstrasse $1+3,3012$ Bern, Switzerland

${ }^{d}$ Department of Terrestrial Magnetism, Carnegie Institution for Science, 5241 Broad Branch Road NW, Washington DC 20015 USA

${ }^{e}$ Department of Geology and Geophysics, Indian Institute of Technology Kharagpur, 721302, Kharagpur, India

${ }^{f}$ Czech Geological Survey, Klárov 3, 11821 Prague 1, Czech Republic

${ }^{g}$ Geowissenschaftliches Zentrum, Georg-August-Universität, Goldschmidtstraße 1, 37077 Göttingen, Germany

Corresponding author: Tel. +1 (301) 4052707

Email address: kberming@umd.edu

Word count: 7314 (excluding Abstract, references, figures, tables) 


\section{ABSTRACT}

Several nucleosynthetic processes contributed material to the Solar System; however, the relative contributions of each process, the timing of their input into the solar nebula, and how well these components were homogenized in the solar nebula remain only partially constrained. The $\mathrm{Ba}$ isotope system is particularly useful in addressing these issues because Ba contains isotopes are synthesized through three nucleosynthetic processes ( $s-, r-, p$-process). In this study, high precision $\mathrm{Ba}$ isotope analyses of 22 different whole rock chondrites and achondrites (carbonaceous chondrites, ordinary chondrites, enstatite chondrites, Martian meteorites, and eucrites) were performed to constrain the distribution of $\mathrm{Ba}$ isotopes on the regional scale in the Solar System. A melting method using aerodynamic levitation and $\mathrm{CO}_{2}$-laser heating was used to oxidize $\mathrm{SiC}$, a primary carrier of $\mathrm{Ba}$ among presolar grains in carbonaceous chondrites. Destruction of these grains during the fusion process enabled the complete digestion of these samples. The Ba isotope data presented here are thus the first for which complete dissolution of the bulk meteorite samples was certain. Enstatite chondrites, ordinary chondrites, and all achondrites measured here possess $\mathrm{Ba}$ isotope compositions that are not resolved from the terrestrial $\mathrm{Ba}$ isotope composition. Barium isotope anomalies are evident in most of the carbonaceous chondrites analyzed, but the ${ }^{135} \mathrm{Ba}$ anomalies are generally smaller than previously reported for similarly sized splits of CM2 meteorites. Variation in the size of the ${ }^{135} \mathrm{Ba}$ anomaly is also apparent in fused samples from the same parent body (e.g., CM2 meteorites) and in different pieces from the same meteorite (e.g., Orgueil, CI). Here, we investigate the potential causes of variability in ${ }^{135} \mathrm{Ba}$, including the contribution of radiogenic ${ }^{135} \mathrm{Ba}$ from the decay of ${ }^{135} \mathrm{Cs}$ and incomplete homogenization of the presolar components on the $<0.8 \mathrm{~g}$ sample scale.

\section{Key words}

Barium isotopes, nucleosynthetic isotope anomaly, ${ }^{135} \mathrm{Cs}-{ }^{135} \mathrm{Ba}$, Solar System evolution. 


\section{INTRODUCTION}

A number of processes can cause isotope ratios in Solar System materials to differ from bulk terrestrial isotope ratios. These processes include (i) radioactive decay (e.g., Reynolds, 1960, 1967; Rowe and Kuroda, 1965), (ii) nuclear reactions following interaction with cosmic rays (e.g., Arnold, 1961), (iii) mass-dependent and -independent isotope fractionation during chemical reactions (e.g., Keesom and van Dijk, 1931; Urey et al., 1932a,b; Thiemens and Heidenreich, 1983; Fujii et al., 2009), and (iv) incomplete mixing in the solar nebula of nucleosynthetic material having exotic isotope abundances from different stellar sources, often referred to as nucleosynthetic isotope anomalies (e.g., presolar grains; Zinner, 1998; Hidaka et al., 2003; Andreasen and Sharma, 2007; Carlson et al., 2007). The non-solar isotope compositions of individual presolar grains (e.g., nucleosynthetic material) result from nucleosynthetic reactions that were active in their stellar source regions (Burbidge et al., 1957). Ion microprobe (SHRIMP-RG) analyses of the Ba isotopic composition of a SiC-enriched bulk sample (KJB) from Murchison (Ávila et al., 2013) show large anomalies in $\delta^{134} \mathrm{Ba} /{ }^{136} \mathrm{Ba}(140 \pm$ 13), $\delta^{135} \mathrm{Ba} /{ }^{136} \mathrm{Ba}(-423 \pm 2), \delta^{137} \mathrm{Ba} /{ }^{136} \mathrm{Ba}(-284 \pm 5)$, and $\delta^{138} \mathrm{Ba} /{ }^{136} \mathrm{Ba}(-281 \pm 23)$, where $\delta{ }^{\mathrm{i}} \mathrm{Ba} /{ }^{136} \mathrm{Ba}=\left[\left({ }^{\mathrm{i}} \mathrm{Ba} /{ }^{136} \mathrm{Ba}\right)_{\text {measured }} /\left({ }^{\mathrm{i}} \mathrm{Ba} /{ }^{136} \mathrm{Ba}\right)_{\text {solar }}-1\right] \times 10^{3}$ (Fig. 1). This isotope signature is generally considered to reflect the $s$-process in Asymptotic Giant Branch (AGB) stars (Savina et al., 2003; Marhas et al., 2007; Ávila et al., 2013). Complementary coupled excesses in ${ }^{135} \mathrm{Ba}$ and ${ }^{137} \mathrm{Ba}$ and have been identified in carbonaceous chondrites and acid leachates (Hidaka et al., 2001; Hidaka et al., 2003; Andreasen and Sharma, 2007; Carlson et al., 2007; Hidaka and Yoneda, 2011; Qin et al., 2011;). The cause of the variations in ${ }^{135} \mathrm{Ba}$ and ${ }^{137} \mathrm{Ba}$ isotope abundances in whole rock meteorites is most frequently attributed to the heterogeneous distribution of presolar grains. This type of isotope anomaly is here termed "nucleosynthetic isotope anomaly" and is the focus of this contribution, which investigates the degree and cause of $\mathrm{Ba}$ isotopic heterogeneity in the solar nebula.

Determining the scale of isotope heterogeneity in the solar nebula is an important aspect of cosmochemistry. These data can be used to determine the number and type of supernova contributors to the Solar System, to test the assumption of isotopic homogeneity of the parent isotope that underpins the use of chronometers based on short- and long-lived radioisotopes, and potentially to trace chemical variability in the nebula that might have existed at during 
planetesimal and planet formation. Published ages may be inaccurate if the initial parent/reference isotope ratio (e.g., ${ }^{135} \mathrm{Cs} /{ }^{133} \mathrm{Cs},{ }^{26} \mathrm{Al} /{ }^{27} \mathrm{Al}$ ) had varied throughout the Solar System (e.g., Makide et al., 2011). Such variations in initial abundances could manifest themselves as nucleosynthetic isotope anomalies in bulk meteorite samples. If significant bulk sample nucleosynthetic anomalies were indisputably identified, then the current Solar System chronometers that utilize these isotopes would have to be replaced by regional chronometers that are based on the local abundances of the parent nuclides.

How well presolar grains were homogenized throughout the Solar System on the asteroidal- and planetary scales remains unclear. The primary reason for the uncertainty on this point is the difficulty in obtaining whole rock chemical analyses of meteorite samples (e.g., carbonaceous chondrites) that contain varying proportions of acid-insoluble components. Regarding the $\mathrm{Ba}$ isotope system, the concern is that the documented coupled excesses in ${ }^{135} \mathrm{Ba}$ and ${ }^{137} \mathrm{Ba}$ in carbonaceous chondrites are the result of incomplete dissolution of presolar $\mathrm{SiC}$ grains in the bulk sample (e.g., Andreasen and Sharma, 2007; Carlson et al., 2007). Presolar SiC grains display large deficits in ${ }^{135} \mathrm{Ba}$ and ${ }^{137} \mathrm{Ba}$ isotopes and failure to digest part of this component can result in a complementary enrichment pattern in ${ }^{135} \mathrm{Ba}$ and ${ }^{137} \mathrm{Ba}$, similar in magnitude to the anomalies that are observed in whole rock carbonaceous chondrites. The fact that differentiated and high metamorphic grade meteorites, which no longer contain intact presolar grains, do not display $\mathrm{Ba}$ isotope anomalies lends support to this concern.

To evaluate the distribution of Ba isotopes in the Solar System, 22 bulk meteorites from different meteorite classes were analyzed in this study while taking special care to ensure complete dissolution of insoluble phases by using a high temperature laser fusion method. Barium is a moderately refractory element $\left(\mathrm{T}_{\mathrm{c}}=1455 \mathrm{~K}, \mathrm{BaTiO}_{3}\right.$; Lodders, 2003; $\mathrm{T}_{\mathrm{c}}=$ temperature at which $50 \%$ of the element has condensed from the solar nebula) and is a trace element in chondrites (CI $=2.34$ ppm; Anders and Grevesse, 1989) with carrier phases including melilite, feldspar, and $\mathrm{SiC}$ (estimates of the Ba concentration in individual SiC grains range from 2-10 ppm; Ávila et al., 2013). Barium has seven stable isotopes that sample three different nucleosynthetic processes ( $s$-, $r$-, and $p$-processes, Table 1). Low thermal neutron capture cross sections of all Ba isotopes require long irradiation times by cosmic rays to create significant changes in $\mathrm{Ba}$ isotope abundances (Table 1; Mughabghab 2003). There is no reported evidence that indicates secondary 
nuclear processes produced shifts in $\mathrm{Ba}$ isotope abundances in cosmochemical samples. Modification of $\mathrm{Ba}$ isotopic composition could also arise from the decay of radioactive species. For example, positive anomalies in ${ }^{135} \mathrm{Ba}$ could result from the decay of the short-lived radioisotope ${ }^{135} \mathrm{Cs}\left(\mathrm{t}_{1 / 2}=2.3 \mathrm{Ma}\right.$; Hidaka et al., 2001; Hidaka and Yoneda, 2011; Hidaka and Yoneda, 2013; Bermingham et al., 2014). Lanthanum-138 decays to ${ }^{138} \mathrm{Ba}$ with a half-life of $\sim 105 \mathrm{Ga}$ (Nakai et al., 1986). Because of the very low natural abundance of ${ }^{138} \mathrm{La}(<0.09 \%)$ and its long half-life, radiogenic ${ }^{138} \mathrm{Ba}$ is only a very minor component in bulk meteorites (Ingham et al., 1947; Anders and Grevesse, 1989).

\section{ANALYTICAL TECHNIQUES}

\subsection{Meteorite samples}

A total of 25 samples (of 22 meteorites) from different classes (carbonaceous chondrites, low and high metamorphic grade $\mathrm{H}$ chondrites, eucrites, and Martian meteorites) were selected for analysis (Table 2). This collection of samples was carefully chosen to determine the Ba isotope composition of material that is as representative of the asteroid belt, Mars, and Earth as possible. To confirm that the methods used for the isolation of $\mathrm{Ba}$ and measurement of its isotopic composition did not impart systematic biases, the $\mathrm{Ba}$ isotope composition of terrestrial basalt (DU17) and two eucrites (Juvinas and Stannern) were measured. Eucrites are not considered to possess nucleosynthetic anomalies (Andreasen and Sharma, 2007) and, therefore, provide an additional robust test for isotope fractionation effects during sample dissolution, ion-exchange chromatographic separation of $\mathrm{Ba}$, and measurement by thermal ionization mass spectrometry (TIMS).

Nearly all (19) of samples selected for this study are observed falls without evidence for terrestrial weathering. Meteorite finds were avoided because such samples, especially those from hot deserts, can contain large amounts of secondary barite. Barite forms by the interaction of brines with a meteorite during the humid seasons (Lee and Bland, 2004), which may make most of the $\mathrm{Ba}$ in such samples terrestrial in origin and unsuitable for this study. Three Martian meteorites that are desert finds (Los Angeles 001, Miller Range 03346, and North West Africa 4864) were included in the sample set. Importantly, however, barite has not been reported in 
these samples (Rubin et al., 2000; Day et al., 2006). The analyzed chips of these samples were taken from the center of the respective meteorites to avoid potential terrestrial alteration products.

\subsection{Sample preparation}

Meteorites were cut using a water-cooled saw with a diamond-coated blade to isolate volumes free of fusion crust or rust patches. Saw marks were removed using corundum paper. After cutting, samples were immersed in water and treated with ultrasound for 20 minutes. Samples were dried, crushed to sand-sized fragments using a steel piston and a hammer, and then ground by hand into a fine powder using an agate mortar and pestle. In this study, sample sizes between 0.05 and $0.95 \mathrm{~g}$ were used. Water used throughout sample preparation, digestion, and column chemistry was deionized with a resistivity of $18.2 \mathrm{M} \Omega \mathrm{cm}$.

\subsection{Laser fusion}

Obtaining accurate whole rock chemical analyses of carbonaceous chondrites has been difficult because of the presence of acid insoluble components, in particular presolar SiC (Hidaka et al., 2003; Andreasen and Sharma, 2007; Carlson et al., 2007; Hidaka and Yoneda, 2011). A reliable laboratory method for dissolving the chemically robust moissanite $(\mathrm{SiC})$ is through oxidation $\left(\mathrm{SiC}+2 \mathrm{O}_{2} \rightarrow \mathrm{SiO}_{2}+\mathrm{CO}_{2}\right)$ via melting the sample in an oxidizing environment. An experimental melting method using an aerodynamic levitation combined with $\mathrm{CO}_{2}$-laser heating at the Geoscience Center at the University of Göttingen, Germany, was used in this study to induce oxidation of $\mathrm{SiC}$ (for experimental details, refer to Pack et al., 2010). All carbonaceous chondrites and low metamorphic grade samples were melted by heating $\sim 10 \mathrm{mg}$ aliquots of the powdered sample (total sample mass ranged from 0.05 to $0.95 \mathrm{~g}$; Table 2) under the laser for 5 to 10 s. The laser heating time and desirable sample mass were determined from earlier experiments using synthetic SiC powders (grain sizes from 3 to $17 \mu \mathrm{m}$ ). A random selection of these and sample melt beads were analyzed using a Scanning Electron Microscope (SEM) Leo 1485 (University of Göttingen) to ensure that complete melting of the aliquot had occurred. Complete melting is indicated by a quenched texture as shown in Fig. 2. Subsequently, melt beads were placed in an ultrasonic water bath for 10 minutes to remove adhering material and dissolved using concentrated acids as described below. 


\subsection{Sample digestion}

Meteorite samples processed at the Westfälische Wilhelms-Universität (WWU) were digested in a 5:1 mixture of $29 \mathrm{M} \mathrm{HF}-14 \mathrm{M} \mathrm{HNO}_{3}$ in sealed, non-pressurized Savillex ${ }^{\circledR}$ vials at $150{ }^{\circ} \mathrm{C}$ on a hotplate for 2 - 5 days. After evaporation, a second attack with concentrated $\mathrm{HF}-\mathrm{HNO}_{3}$ was carried out at $150{ }^{\circ} \mathrm{C}$ for two or four days. Solutions were evaporated with $1-2 \mathrm{ml}$ of concentrated $\mathrm{HClO}_{4}\left(\right.$ Suprapur $\left.{ }^{\circledR}, 70 \%\right)$. Vials containing the sample and an additional $1-2 \mathrm{ml}$ $\mathrm{HClO}_{4}$ were capped and left overnight at $120{ }^{\circ} \mathrm{C}$. Samples were dried down a second time. At this point, the majority of samples consisted of brown solids with no visible white precipitates. For samples in which white precipitates (presumably fluorides) remained, the $\mathrm{HClO}_{4}$ treatment was repeated until the white precipitates were gone. Samples were then dissolved in $10 \mathrm{ml}$ of 6 $\mathrm{M} \mathrm{HCl}$ and left overnight at $120^{\circ} \mathrm{C}$ in capped vials. The samples were then dried down, dissolved in $\sim 5 \mathrm{ml}$ of $14 \mathrm{M} \mathrm{HNO}_{3}$, dried down, and taken up in the loading solution.

A subset of samples was processed at the Department of Terrestrial Magnetism, Carnegie Institution for Science (DTM). These samples were digested as outlined in Carlson et al., (2007), which is essentially the same digestion technique as that used at WWU (with the exception that $\mathrm{HClO}_{4}$ is not used) and thus is not expected to produce systematic differences between the datasets.

All digested samples produced a clear solution, except for Butsura, Ösede, and Allende ${ }_{\text {powder }}$ (Allende $e_{\text {acid }}$ is an aliquot of Allende that did not undergo laser fusion). Butsura and Ösede had a small amount of fine-grained black residue that was identified by X-ray diffraction as Cr-spinel. The Ba abundance in Cr-spinel is negligible (e.g., Grégoire et al., 2000), thus loss of Ba due to incomplete dissolution of this phase is not expected. There was a substantial amount of very finegrained black residue observed in Allende $_{\text {acid, }}$ which is considered to be a mix of organic compounds and insoluble presolar material.

\subsection{Chemical separation of Ba, REE, and major elements}

At WWU, a chemical separation method derived from previous studies was used to separate and isolate Ba, REE, and the major elements (Eugster et al., 1969; Andreasen and Sharma, 2007; Carlson et al., 2007). The first step of this two-stage cation-exchange chemistry uses standard procedures (1-cm diameter x $20 \mathrm{~cm}$ long quartz glass columns filled with AG50W-X8 cation- 
exchange resin, equilibrated in water) with $2 \mathrm{M} \mathrm{HNO}_{3}, 2.5 \mathrm{M}$ and $6 \mathrm{M} \mathrm{HCl}$ as eluents to isolate REE and Ba and major elements, respectively. The same columns were used for the second step, but were cleaned before use and $2.5 \mathrm{M}$ and $6 \mathrm{M} \mathrm{HCl}$ were used as the eluents to separate $\mathrm{Ba}$ from the major elements.

The digested meteorite samples were redissolved in $2 \mathrm{M} \mathrm{HNO}_{3}$ and centrifuged before column chemical separation of the desired elements. Samples were loaded onto the first column in $12 \mathrm{ml}$ of $2 \mathrm{M} \mathrm{HNO}_{3}$. Columns were rinsed with $26 \mathrm{ml}$ of $2 \mathrm{M} \mathrm{HNO}_{3}$ to elute a $\mathrm{Ba}$ and major element cut for processing through a second column. The REE cut was eluted in $18 \mathrm{ml}$ of $6 \mathrm{M} \mathrm{HCl}$. For the second step chemistry, the Ba and major element cut was dried down, dissolved in $6 \mathrm{ml}$ of $2.5 \mathrm{M} \mathrm{HCl}$, and then loaded onto the column. The major element fraction was eluted in $20 \mathrm{ml}$ of $2.5 \mathrm{M} \mathrm{HCl}$ and the $\mathrm{Ba}$ fraction was eluted with $24 \mathrm{ml}$ of $6 \mathrm{M} \mathrm{HCl}$. The $\mathrm{Ba}$ yield from the combined column chemistries was $70-80 \%$.

The method of chemical separation of Ba, REE, and major elements used at DTM is the same as that reported in Carlson et al. (2007). This isolation process is very similar to that used at WWU and is not expected to produce systematic differences between the datasets.

The total procedural Ba blank for non-fused samples processed at WWU and DTM was 0.2 - 1 ng. The amount of Ba blank stemming from the laser fusion was estimated by processing ca. 0.5 $\mathrm{g}$ of powdered gem-quality quartz of known $\mathrm{Ba}$ concentration. After correcting for the $\mathrm{Ba}$ contribution of the quartz, the total blank arising from the melting of a sample was $5 \mathrm{ng}$ or less, which typically corresponds to $0.3 \%$ or less of the total $\mathrm{Ba}$ processed. The total procedural blanks for fused samples (5.2 - $6 \mathrm{ng})$ and non-fused samples (0.2 - $1 \mathrm{ng})$ are insignificant, thus blank corrections were not applied.

\subsection{Isotope measurements}

Barium isotope data were collected during different measurement campaigns at WWU and DTM using a Thermo Fisher TIMS. Almost identical loading and measurement methods were used at DTM and WWU, so a systematic difference between the datasets resulting from the loading and measurement techniques is unlikely. 
At WWU, a double filament assembly (Ta-evaporation, Re-ionization) was used because it produced more stable signals and thus more reproducible measurements of a $\mathrm{Ba}$ standard solution than a single Re filament. The separated $\mathrm{Ba}$ was dissolved in $6 \mathrm{M} \mathrm{HCl}$ and, just before loading, $\sim 1 \mu \mathrm{L} 0.04 \mathrm{M} \mathrm{H}_{3} \mathrm{PO}_{4}$ was mixed into this solution.

The TIMS at WWU is equipped with nine Faraday collectors enabling ${ }^{132,134-138} \mathrm{Ba}$ and interference monitors ${ }^{139} \mathrm{La}$ and ${ }^{140} \mathrm{Ce}$ to be measured using the static mode. Barium-130 was not measured because the tether connecting L4 and L3 was too short to allow simultaneous measurement of ${ }^{130} \mathrm{Ba}$ and ${ }^{132} \mathrm{Ba}$. Generally, the ${ }^{132} \mathrm{Ba} /{ }^{136} \mathrm{Ba}$ values have large analytical uncertainties (ca. $220 \mathrm{ppm}$ ) associated with their measurement because of the low natural abundance of the $p$-process isotope ${ }^{132} \mathrm{Ba}(\sim 0.1 \%)$. As a result of the poor analytical precision, only upper limits can be given for the $p$-process contribution. The analyses were carried out in static mode rather than dynamic mode because all isotopes (except ${ }^{130} \mathrm{Ba}$ ) could be measured in 2 hours (200 - $400 \mathrm{ng}$ Ba load produce a ${ }^{138} \mathrm{Ba}$ intensity of $\sim 20 \mathrm{~V}$ for $\sim 2$ hours; 18 blocks of 18 ratios, $\sim 320$ integrations per filament). The alternative dynamic mode that was considered would have significantly increased the measurement time, which may have led to detrimental sample fractionation effects (e.g., Roth et al., 2014) and filament reservoir mixing effects (e.g., Upadhyay et al., 2008; Andreasen and Sharma 2009). Barium isotope data were collected during four different analytical sessions, which were each separated by $\sim 4$ months. Because long-term drift in Faraday cup performance does not cancel out in static analyses as it can in multi-dynamic analyses, the samples were referenced only to the standard data collected within their respective analytical session. For this reason, the reported external precision varies among the analytical sessions (Tables 3 - 6; Figs. 3 and 4). Peak centering and lens focusing were performed every fourth block. The amplifier connections were rotated after each block and baselines were measured at the beginning of each block. Isobaric interferences from ${ }^{138} \mathrm{La}$ and ${ }^{136,138} \mathrm{Ce}$ were monitored using ${ }^{139} \mathrm{La}$ and ${ }^{140} \mathrm{Ce}$ but were found to be at baseline levels during all measurements, thus no interference corrections were applied. For details of TIMS data acquisition at DTM, see Carlson et al. (2007). Notably, ${ }^{130} \mathrm{Ba}$ and ${ }^{132} \mathrm{Ba}$ were both measured during the analytical campaign at DTM because the tether connecting L4 and L3 was long enough to allow simultaneous measurement of ${ }^{130} \mathrm{Ba}$ and ${ }^{132} \mathrm{Ba}$. 
The $\mathrm{Ba}$ from each sample digestion was measured up to 4 times, using new Ba-loads on new filaments. Sample data were compared to terrestrial basalt (DU17) data and a Ba standard solution (Specpure ${ }^{\circledR}$ Alfa Aesar). Multiple analyses of the Ba standard solution yielded precisions better than $\pm 12 \mathrm{ppm}, \pm 29 \mathrm{ppm}$, and $\pm 11 \mathrm{ppm}$ for ${ }^{135} \mathrm{Ba} /{ }^{138} \mathrm{Ba},{ }^{136} \mathrm{Ba} /{ }^{138} \mathrm{Ba}$, and ${ }^{137} \mathrm{Ba} /{ }^{138} \mathrm{Ba}$, respectively, as determined from the $2 \sigma$ of the population of replicate standard measurements (see Tables 3 - 6).

All data presented here have been corrected for mass fractionation using the exponential law referenced to ${ }^{134} \mathrm{Ba} /{ }^{138} \mathrm{Ba}=0.03371$ (De Bièvre and Taylor, 1993; Andreasen and Sharma, 2007). Previous studies commonly used ${ }^{134} \mathrm{Ba} /{ }^{136} \mathrm{Ba}$ (s-process only isotopes) for nominalization (Hidaka et al., 2003; Andreasen and Sharma, 2007; Carlson et al., 2007). In this study, ratios involving ${ }^{136} \mathrm{Ba}$ showed unexpectedly poor reproducibility, which would be propagated into all ratios if the data were fractionation corrected to ${ }^{134} \mathrm{Ba} /{ }^{136} \mathrm{Ba}$. We found an improvement in ratio precision ranging from a factor of 4 on ${ }^{135} \mathrm{Ba}$ and 2 on ${ }^{137} \mathrm{Ba}$ when fractionation correcting instead to ${ }^{134} \mathrm{Ba} /{ }^{138} \mathrm{Ba}$. The cause of the lowered precision on ${ }^{136} \mathrm{Ba}$ is not clear, but we suspect degradation of the axial faraday cup as this phenomenon was not observed in the $\mathrm{Ba}$ data collected on the same instrument, using essentially the same techniques, as reported by Carlson et al. (2007).

An advantage of normalizing to ${ }^{134} \mathrm{Ba} /{ }^{138} \mathrm{Ba}$ is that it covers a larger spread in masses and thus yields a more precise measure of the isotope fractionation during the analysis. As long as there is no significant contribution from ${ }^{139} \mathrm{La}$ to ${ }^{138} \mathrm{Ba}$, this normalization scheme is appropriate for cosmochemical samples. The $\mathrm{La} / \mathrm{Ba}$ was monitored and was found to be chondritic or subchondritic in all samples, thus indicating that referencing data to ${ }^{134} \mathrm{Ba} /{ }^{138} \mathrm{Ba}$ is a sound approach. All data are presented using the $\mu$ notation (deviations in parts per million from the terrestrial standard) for comparison between present and published data.

\section{RESULTS}

\subsection{Barium isotope distribution in whole rock meteorite samples}


The Ba isotope compositions of DU17, Juvinas, and Stannern were found to be analytically indistinguishable from those of the Ba standard measured during this study (Table 3, Fig. 3). The data for DU17 and Stannern, and the replication of reported Ba isotopic composition of Juvinas, indicate that the methods of digestion, chemical separation, and isotope measurement used do not impart biases. The $\mathrm{Ba}$ isotope compositions of Martian meteorites, eucrites, enstatite and ordinary chondrites fall within the external precision of the Ba standard solution indicating that these samples have $\mathrm{Ba}$ isotope compositions indistinguishable from terrestrial values (Tables 3 5; Fig. 4). The absence of Ba isotope anomalies in these meteorites concurs with published data of meteorites from the same groups (Hidaka et al., 2003; Andreasen and Sharma, 2007; Carlson et al., 2007). Excesses in ${ }^{135} \mathrm{Ba}$ and ${ }^{137} \mathrm{Ba}$ are analytically resolvable from the $\mathrm{Ba}$ standard in Orgueil, Allende, Vigarano, Cold Bokkeveld, Lancé, and Kainsaz (Tables 5 - 6 and Fig. 4). The Ba isotope data from Renazzo (CR2), Murchison (CM2), and Mighei (CM2) do not have isotope anomalies that are well resolved from the terrestrial standard.

The $\mathrm{Ba}$ isotope anomalies in the studied carbonaceous chondrites are generally similar to those reported by Hidaka et al. (2003), Andreasen and Sharma (2007), and Carlson et al. (2007). When comparing our fused meteorite data with published data (unfused) from the same sample, however, there is a smaller ${ }^{135} \mathrm{Ba}$ anomaly in CM2 samples that underwent fusion, despite similar levels of precision reached in the relevant studies (Table 7). For example, data from the present study for Cold Bokkeveld shows excesses in ${ }^{135} \mathrm{Ba} /{ }^{138} \mathrm{Ba}$ of $34 \pm 7$ ppm whereas Carlson et al., (2007) reported ${ }^{135} \mathrm{Ba} /{ }^{138} \mathrm{Ba}=53 \pm 11 \mathrm{ppm}$ anomalies (the ${ }^{134} \mathrm{Ba} /{ }^{138} \mathrm{Ba}$-normalized data were obtained from unpublished data collected as part of the Carlson et al. 2007 study). The Ba isotope data for Mighei collected in our study do not possess resolvable ${ }^{135} \mathrm{Ba}$ and ${ }^{137} \mathrm{Ba}$ anomalies. This differs from the results from Carlson et al. (2007), who reported a small ${ }^{135} \mathrm{Ba} /{ }^{138} \mathrm{Ba}$ excess in Mighei (24 $\left.\pm 11 \mathrm{ppm}\right)$.

A slight reduction in ${ }^{135} \mathrm{Ba} /{ }^{138} \mathrm{Ba}$ variability, however, is not evident when comparing our $\mathrm{Ba}$ isotope compositions obtained on fused pieces of Orgueil (CI) to published data on unfused samples of the same meteorite. Harper et al., (1991) published two compositions for Orgueil $\left({ }^{135} \mathrm{Ba} /{ }^{136} \mathrm{Ba}\right.$ of $20 \pm 7$ and $\left.40 \pm 8 \mathrm{ppm}\right)$. Carlson et al., (2007) reported a ${ }^{135} \mathrm{Ba} /{ }^{138} \mathrm{Ba}$ value of $31 \pm 11$ ppm, which is between those of two different fused pieces studied here (Orgueil 1: $53 \pm 12 \mathrm{ppm}$, and Orgueil 2: 22 \pm 7 ppm; Fig. 4c,d; Table 7). 
Closer examination of the $\mathrm{Ba}$ isotope compositions of fused samples in this study reveals a notable variation in the magnitude of the ${ }^{135} \mathrm{Ba} /{ }^{138} \mathrm{Ba}$ anomaly between samples from the same group. For example, the Ba isotope composition of samples from the CM2 parent body, Cold Bokkeveld $\left({ }^{135} \mathrm{Ba} /{ }^{138} \mathrm{Ba}=34 \pm 7 \mathrm{ppm}\right)$ and Mighei $\left({ }^{135} \mathrm{Ba} /{ }^{138} \mathrm{Ba}=14 \pm 7 \mathrm{ppm}\right)$, have resolved differences in ${ }^{135} \mathrm{Ba}$ isotope compositions. Variation in ${ }^{135} \mathrm{Ba}$ is also apparent in different pieces of the same meteorite that have undergone fusion (e.g., Orgueil, CI). When comparing the Ba isotope compositions obtained on 2 pieces of Orgueil (both 0.7 - $0.8 \mathrm{~g}$ and fused) obtained from two different museums (NHM UK and Smithsonian USA), a resolvable difference is observed in ${ }^{135} \mathrm{Ba} /{ }^{138} \mathrm{Ba}$ (Orgueil 1: 53 \pm 12 ppm; Orgueil 2: $22 \pm 7$ ppm).

\section{DISCUSSION}

\subsection{Ba isotope distribution in the Solar System}

The coupled excesses in ${ }^{135} \mathrm{Ba}$ and ${ }^{137} \mathrm{Ba}$ identified here in carbonaceous chondrites generally concur with previous studies (Hidaka et al., 2003; Andreasen and Sharma, 2007; Carlson et al., 2007). Fused samples from the CM2 group have slightly smaller Ba isotope anomalies than the same samples processed only by acid dissolution. The magnitude of the ${ }^{135} \mathrm{Ba} /{ }^{138} \mathrm{Ba}$ anomaly varies among fused samples from the same parent body (e.g., Cold Bokkeveld and Mighei; CM2 meteorites) and also among different pieces of the same meteorite (e.g., Orgueil 1 and Orgueil 2; $\mathrm{CI}$ ). This small but resolvable variation in ${ }^{135} \mathrm{Ba}$ is striking because it is generally assumed that meteorites originating from the same parent body possess the same nucleosynthetic isotope composition, as long as the isotope system in question is not susceptible to modification via secondary reactions, such as cosmic ray exposure effects (e.g., Wittig et al., 2013) or radioactive decay. The CRE ages of samples studied here are all $\leq 41$ Ma (data from Pepin et al., 1966; Heymann and Anders, 1967; Marti and Graf, 1992; Bischoff et al., 1993; Bischoff et al., 1996; Schultz and Weber 1996; Herzog et al., 1997; Mazor et al., 1998; Miura et al., 1998; Terribilini et al., 2000; Eugster et al., 2002; Huss et al., 2008; Roth et al., 2011; Table 2) and are not old enough to produce the resolved $\mathrm{Ba}$ isotope variations via neutron capture reactions.

The variation in ${ }^{135} \mathrm{Ba} /{ }^{138} \mathrm{Ba}$ compositions of samples that were fused from those that were not fused, and among fused samples of the same meteorite groups/meteorite is unlikely to stem from 
neutron capture effects because all $\mathrm{Ba}$ isotopes have low neutron capture cross sections (Table 1). The development of a positive ${ }^{135} \mathrm{Ba}$ anomaly could potentially occur via a neutron reaction pathway from neighboring nuclides, but such a pathway is currently unknown. The Ba isotope anomaly variations are also unlikely to originate from nuclear field effects because the pattern of the observed ${ }^{135} \mathrm{Ba}$ and ${ }^{137} \mathrm{Ba}$ anomalies does not match the pattern expected for massindependent fractionation (Fujii et al., 2009). Instead, these effects could related to: (1) nucleosynthetic or radiogenic variation in the abundance of ${ }^{138} \mathrm{Ba}$ used in the normalization ratio; (2) the digestion techniques employed in the respective studies; (3) contribution of radiogenic

${ }^{135} \mathrm{Ba}$; or (4) incomplete homogenization of the presolar or refractory component at the sampling scale of this and published studies.

\subsection{Potential effects of nucleosynthetic or radiogenic ${ }^{138} \mathrm{Ba}$ anomalies}

In the present study, all data were corrected for mass fractionation using the exponential law with ${ }^{134} \mathrm{Ba} /{ }^{138} \mathrm{Ba}=0.03371$ as the reference ratio (De Bièvre and Taylor, 1993; Andreasen and Sharma, 2007). This ratio comprises isotopes that were produced via different nucleosynthetic processes ( $s$-only and $s+r$-processes, respectively) and potentially radiogenic ${ }^{138} \mathrm{Ba}$ from the decay of the rare ${ }^{138} \mathrm{La}$. Nucleosynthetic isotope anomalies or radiogenic contributions to these normalizing ratios can thus potentially result in propagation of ${ }^{138} \mathrm{Ba}$ variability into the other $\mathrm{Ba}$ isotope ratios through the fractionation correction. Isotope anomalies in ${ }^{138} \mathrm{Ba}$ have been measured in the acid residue of Murchison (Qin et al., 2011) and in pre-solar SiC grains (Pellin et al., 2000). Generally, these isotope variations have been attributed to s-process variability. Direct evidence for nucleosynthetic variation in ${ }^{138} \mathrm{Ba}$ on the whole rock sample scale, however, is yet to be found. The coupled excesses in ${ }^{137} \mathrm{Ba}$ and ${ }^{138} \mathrm{Ba}$ reported by Ranen and Jacobsen (2006) were initially explained by incomplete mixing of neutron burst material in the early solar nebula, but were later shown to result from an analytical artifact (Andreasen and Sharma, 2007). The absence of nucleosynthetic $\mathrm{Ba}$ isotope variations suggests that if there are anomalous nucleosynthetic contributions to ${ }^{138} \mathrm{Ba}$, they are smaller than the current analytical uncertainty achievable with state-of-the-art mass spectrometers and, therefore, would not adversely affect the mass fractionation corrected data.

Evidence for the decay of ${ }^{138} \mathrm{La}$ to ${ }^{138} \mathrm{Ba}$ has recently been suggested to be the source of ${ }^{138} \mathrm{Ba}$ variations identified in three Group II CAIs (Brennecka et al., 2013) and could also potentially 
affect ${ }^{134} \mathrm{Ba} /{ }^{138} \mathrm{Ba}$-normalized data. The potential contribution from ${ }^{138} \mathrm{La}$ can be constrained by obtaining $\mathrm{La} / \mathrm{Ba}$ for those samples with anomalous $\mathrm{Ba}$ isotope compositions and contrasting it with chondritic $\mathrm{La} / \mathrm{Ba} \sim 0.1$ (Lodders, 2003). An aliquot of the carbonaceous chondrites processed at DTM was removed before column chemistry and analyzed using an $i C A P Q$ ICPMS (ThermoFisher) housed at DTM to obtain whole rock elemental abundances. These carbonaceous chondrites have chondritic to subchondritic $\mathrm{La} / \mathrm{Ba}(0.09-0.12)$ which constrain ${ }^{138} \mathrm{Ba}^{*} /{ }^{138} \mathrm{Ba}$ to $<4.6 \times 10^{-6}$, where ${ }^{138} \mathrm{Ba}^{*}$ refers to radiogenic ${ }^{138} \mathrm{Ba}$. In order to produce significant radiogenic ${ }^{138} \mathrm{Ba}^{*}\left({ }^{138} \mathrm{Ba}^{*} /{ }^{138} \mathrm{Ba}>1 \times 10^{-5}\right), \mathrm{La} / \mathrm{Ba}$ would have to exceed 0.26 . Thus, there is no significant contribution by ${ }^{138} \mathrm{La}$ for these samples and the variation seen in the magnitude of $\mathrm{Ba}$ isotope anomalies from samples of the same meteorite cannot be attributed to radiogenic ${ }^{138} \mathrm{Ba}$. Another aspect of this issue is that variability only in ${ }^{138} \mathrm{Ba}$ would be propagated into all the isotope ratios, with the magnitude of offset increasing proportional to the mass difference from ${ }^{138} \mathrm{Ba}$. Where anomalous $\mathrm{Ba}$ is observed in the data reported here, the data do not show the massdependent anomalies that would be expected if the anomaly were present only in ${ }^{138} \mathrm{Ba}$.

Additionally, variation in ${ }^{135} \mathrm{Ba}$ among samples from the CM2 parent body (CM2: Cold Bokkeveld ${ }^{135} \mathrm{Ba} /{ }^{136} \mathrm{Ba}=58 \pm 7 \mathrm{ppm}$; Mighei ${ }^{135} \mathrm{Ba} /{ }^{136} \mathrm{Ba}=27 \pm 7 \mathrm{ppm}$; Murray ${ }^{135} \mathrm{Ba} /{ }^{136} \mathrm{Ba}=12 \pm 7$ ppm; CV3: Grosnaja ${ }^{135} \mathrm{Ba} /{ }^{136} \mathrm{Ba}=40 \pm 7 \mathrm{ppm}$ and Mokoia ${ }^{135} \mathrm{Ba} /{ }^{136} \mathrm{Ba}=-12 \pm 7 \mathrm{ppm}$ ) also is evident in the dataset published by Carlson et al., (2007) in which normalization was by the $s$ process only $\left({ }^{134} \mathrm{Ba} /{ }^{136} \mathrm{Ba}\right)$ ratio. This suggests that the source of the ${ }^{135} \mathrm{Ba}$ variation is not related to the choice of normalizing ratio.

\subsection{Digestion techniques}

The pre-digestion laser treatment employed in the present study effectively oxidized $\mathrm{SiC}$, a primary carrier of large Ba isotope anomalies (e.g., Marhas et al., 2007, Ávila et al., 2013) enabling complete sample dissolution. The digestion techniques employed in earlier studies on Ba isotopes in meteorites (Hidaka et al., 2003; Andreasen and Sharma, 2007; Carlson et al., 2007) were designed to dissolve $\mathrm{SiC}$ and other chemically resistant presolar phases, but complete dissolution was not documented. To determine if the pre-digestion laser treatment improved dissolution, two different samples of the Allende Smithsonian Reference Powder were analyzed: (a) Allende $_{\text {fused }}(0.52 \mathrm{~g})$ which was melted with the laser before acid digestion, and (b) Allende $_{\text {acid }}(0.61 \mathrm{~g})$ which underwent acid digestion only. Allende was chosen for this test 
because it contains $\mathrm{SiC}$ that can alter its bulk $\mathrm{Ba}$ isotope composition if not digested (concentration of $\mathrm{SiC}$ in Allende $~ 0.01 \mathrm{ppm}$; Huss, 1995). A fine black residue (assumed to be a mixture of primarily C-bearing compounds and insoluble refractory grains) remained after Allende $_{\text {acid }}$ was treated with two rounds of concentrated $\mathrm{HF}-\mathrm{HNO}_{3}$. In contrast, Allende fused produced a clear solution after two rounds of $\mathrm{HF}-\mathrm{HNO}_{3}$ digestion. The $\mathrm{Ba}$ isotope composition of both Allende (a) and (b) are identical within analytical uncertainty (Fig. 4d), suggesting that there is no significant dependence of the $\mathrm{Ba}$ isotope composition on the digestion method, and that, for Allende, laser fusion is not required to obtain complete dissolution. This conclusion, however, need not apply to other meteorite groups (or isotope systems). The generally smaller magnitude of $\mathrm{Ba}$ isotope anomalies identified in the CM2 chondrites (e.g., Cold Bokkeveld and Mighei) here as compared to those found in previous studies may indicate that incomplete dissolution does slightly increase the magnitude $(\leq 20 \mathrm{ppm})$ of ${ }^{135} \mathrm{Ba}$ anomalies for SiC-rich samples. The similarity in the Allende sample compositions may be because there is so little $\mathrm{SiC}$ in these samples that the effects of laser fusion are not pronounced. Conducting a comparable test using a SiC-rich sample (e.g., Murchison, CM2, SiC + spinel = 1640 ppm; Huss et al., 2003) may clarify this point, however, obtaining large amounts of such samples was not possible during this study.

\subsection{Potential effects of radiogenic ${ }^{135} \mathrm{Ba}$}

Recent studies have discussed the potential contribution to ${ }^{135} \mathrm{Ba}$ compositions in acid leachates and Allende CAIs and from the decay of short lived ${ }^{135} \mathrm{Cs}\left({ }^{135} \mathrm{Ba}_{\text {radiogenic }} \sim 16\right.$ - 48 ppm; Hidaka et al., 2001; Hidaka and Yoneda, 2011; Hidaka and Yoneda, 2013; Bermingham et al., 2014). The variation in ${ }^{135} \mathrm{Ba}$ observed in samples from the same parent body (Cold Bokkeveld and Mighei, CM2) and also in aliquots from the same meteorite (Orgueil) could result from variable contribution of ${ }^{135} \mathrm{Cs}$ decay to a pre-existing coupled ${ }^{135} \mathrm{Ba}$ and ${ }^{137} \mathrm{Ba}$ nucleosynthetic anomaly. If this is the cause of the variation in ${ }^{135} \mathrm{Ba}$, there should be a corresponding variation in the suprachondritic $\mathrm{Cs} / \mathrm{Ba}$ of these samples. For the carbonaceous chondrites samples analyzed here, however, $\mathrm{Cs} / \mathrm{Ba}$ was below detection limit because of Cs loss during laser fusion. Carlson et al., (2007) did not report $\mathrm{Cs} / \mathrm{Ba}$ for their samples. Thus the potential effect of radiogenic ${ }^{135} \mathrm{Ba}$ remains only a possible explanation for the variation in ${ }^{135} \mathrm{Ba}$ observed. 
The quantity of radiogenic ${ }^{135} \mathrm{Ba}$ can be estimated from literature $\mathrm{Cs} / \mathrm{Ba}$ values for whole rock samples of the relevant meteorites. Using the only available published data for the relevant carbonaceous chondrites (Allende, Mighei, Kainsaz, and Vigarano; Ahren et al., 1973; Krähenbühl et al. 1973; Anders et al., 1976; Wolf et al., 1980; Knab, 1981; Becker et al., 1987; Barrat et al., 2012), these samples all have subchondritic (0.01-0.06) Cs/Ba. Prima facie, this may suggest that there is not a radiogenic ${ }^{135} \mathrm{Ba}$ component in these samples. However, using $\mathrm{Cs} / \mathrm{Ba}$ values from the literature for this application is tenuous because $\mathrm{Cs}$ and $\mathrm{Ba}$ can be mobilized by aqueous alteration (Hidaka and Yoneda, 2013; Bermingham et al., 2014). Thus, an accurate assessment of the potential radiogenic ${ }^{135} \mathrm{Ba}$ component can only be made from $\mathrm{Ba}$ isotope data and $\mathrm{Cs} / \mathrm{Ba}$ measured on the same digested material. Moreover, because isotopic excesses of radiogenic ${ }^{135} \mathrm{Ba}$ are often masked by s-process nucleosynthetic anomalies and the $\mathrm{Cs} / \mathrm{Ba}$ of bulk rocks is typically low, identifying isotopic evidence of radiogenic ${ }^{135} \mathrm{Ba}$ on the whole rock meteorite scale is difficult. In future studies, the physical separation of major presolar materials from meteorite samples before digestion might allow more precise estimates of radiogenic ${ }^{135} \mathrm{Ba}$ contributions.

If the variable abundance of ${ }^{135} \mathrm{Ba}$ identified in some whole rock meteorites is a result of a radiogenic ${ }^{135} \mathrm{Ba}$ component, this has important implications. First, it indicates that the CM2 and CI parent bodies formed in the early stages Solar System evolution $(\leq 9$ Myr after CAI formation), that is, within the lifetime of ${ }^{135} \mathrm{Cs}\left(\mathrm{t}_{1 / 2}=2.3 \mathrm{Myr}\right.$; Hidaka et al, 2001). Second, it suggests that significant $\mathrm{Cs} / \mathrm{Ba}$ fractionation, likely during parent body aqueous alteration, also occurred during these early stages. Supporting evidence for the early formation and onset of aqueous alteration (2.4 - 5 Myr after CAI formation) on parent bodies has been identified from Mn-Cr ages for carbonates in CM and CI chondrites (e.g., Fujiya et al., 2012, 2013; Jilly et al., 2014). The range in estimates of the $\left[{ }^{135} \mathrm{Cs} /{ }^{133} \mathrm{Cs}\right]_{0}$ from $1 \times 10^{-5}$ to $2 \times 10^{-3}$ (Hidaka et al., 2001; Nichols et al., 2002; Srinivasan et al., 2011; Hidaka and Yoneda, 2013), coupled with highly variable $\mathrm{Cs} / \mathrm{Ba}$ for a limited number of carbonaceous chondrites (0.0141- 0.082; Rocholl and Jochum, 1993; Hidaka and Yoneda, 2013; Bermingham et al., 2014), makes it very difficult to estimate the potential amount of radiogenic $\mathrm{Ba}$ or meaningful ages for the onset of aqueous alteration. The large ranges in proposed $\left[{ }^{135} \mathrm{Cs} /{ }^{133} \mathrm{Cs}\right]_{0}$ and observed $\mathrm{Cs} / \mathrm{Ba}$ may in fact reflect significant remobilization of $\mathrm{Cs}$ and/or $\mathrm{Ba}$ in the parent body during aqueous alteration (Hidaka and Yoneda, 2013; Bermingham et al., 2014). Obtaining a more precise initial ratio is critical in 
establishing a more precise time for the onset of aqueous alteration on different parent bodies. Development and application of the ${ }^{135} \mathrm{Cs}^{135} \mathrm{Ba}$ system can address outstanding questions regarding the timing of parent body formation and onset of aqueous alteration. Future work should include refinement of the $\left[{ }^{135} \mathrm{Cs} /{ }^{133} \mathrm{Cs}\right]_{0}$ and expansion of the current whole rock meteorite database for which high precision coupled $\mathrm{Ba}$ isotope and $\mathrm{Cs} / \mathrm{Ba}$ data are available.

\subsection{Sample size effect}

The variation in the size of $\mathrm{Ba}$ isotope anomalies between different laser-fused aliquots of the same meteorite may reflect incomplete homogenization of the presolar or refractory component at the $\sim 0.8 \mathrm{~g}$ (mass of Orgueil used in this study) sample scale in these meteorites. The effect of sample heterogeneity on the major and trace element composition of carbonaceous chondrites (e.g., Allende and Orgueil) has been discussed in detail by Morlok et al. (2006) and Stracke et al. (2012). Both studies indicate that there is a mass 'threshold' for the observance of strong chemical variation within single carbonaceous chondrites, with CAIs exerting a disproportionate control on the refractory element budget of a bulk meteorite sample when present in the sample. The 'threshold' at which homogeneous chemical compositions can be obtained has been reported to be about 1 - $2 \mathrm{~g}$ (Morlok et al., 2006).

The mass 'threshold' for isotopic homogeneity, however, has not been discussed widely in the literature. On the basis of our data, measurable variation in the magnitude of ${ }^{135} \mathrm{Ba} /{ }^{138} \mathrm{Ba}$ anomalies is observed in (fused) carbonaceous chondrite samples (specifically Orgueil) that are smaller than $\sim 0.8 \mathrm{~g}$. This variation is unlikely to only reflect an oversampling of refractory inclusions (e.g., CAIs), because the sample for which the most resolvable variation in ${ }^{135} \mathrm{Ba}$ is present (Orgueil), does not contain preserved CAIs. In contrast, the Ba isotope compositions of samples from the CV3 parent body, which have a high proportion of preserved CAIs, are generally more consistent than those of samples from the CM2 or CI parent bodies (Table 7). The cause for the variation in ${ }^{135} \mathrm{Ba}$ in the CM2 and CI fused samples is thus more likely a result of the incomplete heterogeneous distribution of a presolar component (and, potentially a radiogenic component) on the $<0.8 \mathrm{~g}$ scale.

Samples that are $\sim 0.8 \mathrm{~g}$ or less (Cold Bokkeveld, Mighei, Orgueil 1, Orgueil 2) and display variable ${ }^{135} \mathrm{Ba}$ compositions likely indicate that larger samples are required to obtain a 
representative isotope composition. Determining the mass 'threshold' for isotopic homogeneity would best be done by analyzing multiple large aliquots $(1-5 \mathrm{~g})$ of samples rich in presolar material (e.g., Murchison) and comparing the Ba isotope composition of each fraction.

\subsection{Significance of Ba isotope composition in bulk meteorites}

Barium isotope data obtained from different carbonaceous chondrites for which complete dissolution was achieved indicates that there was a degree of heterogeneous distribution of presolar carriers throughout the Solar System. These new $\mathrm{Ba}$ isotope data confirm that the previously reported nucleosynthetic anomalies (Hidaka et al., 2003; Carlson et al., 2007; Andreasen and Sharma, 2007) were not a result of incomplete sample dissolution, although there may be a slight reduction in size of the anomaly with complete digestion. The origin of the coupled excesses in ${ }^{135} \mathrm{Ba}$ and ${ }^{137} \mathrm{Ba}$ likely result from an $s$-process deficit in the feeding zones of these parent bodies, as suggested by Hidaka et al. (2003), Carlson et al. (2007), Andreasen and Sharma (2007). These anomalies may be coupled with a minor radiogenic ${ }^{135} \mathrm{Ba}$ component in some meteorite samples; however, a more complete dataset is required to confirm this proposition.

The absence of resolvable $\mathrm{Ba}$ isotope anomalies in $\mathrm{H}$ chondrites, enstatite chondrites, eucrites, and Martian meteorites indicates that some parts of the Solar System were well mixed with respect to $\mathrm{Ba}$ isotopes. In addition, either ${ }^{135} \mathrm{Cs}$ was not present in these regions or there was insufficient $\mathrm{Cs} / \mathrm{Ba}$ fractionation within the lifetime of ${ }^{135} \mathrm{Cs}$ to create measurable radiogenic $\mathrm{Ba}$ at the bulk sample scale (e.g., H-chondrites; Hidaka et al., 2001). Why some regions of the Solar System were homogenized with respect to presolar carriers for $\mathrm{Ba}$ whereas other regions were not remains an open question. One explanation is that those regions with homogeneous $\mathrm{Ba}$ isotope compositions represent larger and/or better-mixed feeding zones (e.g., Mars and the HED parent body). This would suggest that the presence of nucleosynthetic isotope anomalies on the whole rock meteorite scale in some samples may, in part, reflect a smaller parent body which formed from a less homogeneous feeding zone. Additional high precision Ba isotope data from large samples $(>0.8 \mathrm{~g})$ of carbonaceous chondrites and those samples for which there are currently no $\mathrm{Ba}$ isotope data (e.g., primitive achondrites) would aid in mapping out the $\mathrm{Ba}$ isotope distribution throughout the Solar System. 
The preservation of only small $\mathrm{Ba}$ isotope anomalies on the bulk sample scale indicates that the presolar grain carriers of highly isotopically anomalous Ba were fairly well mixed in the solar nebula, and even completely homogenized in some regions of the disk (e.g., feeding zones of the HED, H-chondrite, and enstatite chondrite parent bodies, and Mars). This well mixed composition is not evident in other isotopic systems, for example $\mathrm{Ti}, \mathrm{Cr}, \mathrm{Mo}$, and $\mathrm{Ru}$, which display much larger $(>100 \mu)$ nucleosynthetic anomalies in bulk meteorites (Trinquier et al., 2009; Qin et al., 2010; Burkhardt et al., 2011; Fischer-Gödde et al., 2015). The preservation of larger anomalies in these elements but not in Ba provides important constraints. It indicates that the carrier(s) of $\mathrm{Ba}$ isotope anomalies were decoupled from those of $\mathrm{Ti}, \mathrm{Cr}, \mathrm{Mo}$, and $\mathrm{Ru}$. This is not unexpected, because studies have indicated that the main carriers of these elements are likely not SiC (e.g., Trinquier et al., 2009; Qin et al., 2010; Burkhardt et al., 2012; Fischer-Gödde et al., 2015). The preservation of only small isotope anomalies in the $\mathrm{Ba}$ isotope system also indicates that the $\mathrm{Ba}$ isotope carriers were better mixed in the solar nebula than those for $\mathrm{Ti}, \mathrm{Cr}$, Mo, and $\mathrm{Ru}$. The mechanism(s) responsible for spatially variable homogenization is open for debate. Viable mechanisms include 1) variable mixing of different presolar carriers within the solar nebula, or 2) emplacement of $\mathrm{Ba}$ isotope carriers into the nebula-forming region before some isotope carriers (e.g., Ti, Cr) thus allowing more time for them to homogenize. Additionally, the $\mathrm{Ba}$ isotope carriers may have been more robust and thus resistant to chemical fractionation thereby escaping thermal processing in the nebula, which imparted isotope variations in some isotope systems (e.g., Ti and Mo; Trinquier et al., 2009; Burkhardt et al., 2012). Clearly more work is required to elucidate the processes responsible for generating the origins of nucleosynthetic anomalies in a number of isotope systems and this can only be done using data for which it is confirmed that there are no adverse effects of incomplete sample dissolution.

\section{CONCLUSIONS}

This study reports the first $\mathrm{Ba}$ isotope compositions for carbonaceous chondrites for which complete dissolution was certain. Resolvable anomalies in ${ }^{135} \mathrm{Ba}$ and ${ }^{137} \mathrm{Ba}$ are identified in most of these samples and are generally similar to the previously reported $\mathrm{Ba}$ isotope compositions from carbonaceous chondrites for which complete chemical digestion may not have been achieved. The common slight reduction in magnitude of $\mathrm{Ba}$ isotope anomalies in fused samples 
from the CM2 group as compared to compositions of non-fused samples reported in Carlson et al. (2007) may indicate that incomplete dissolution of presolar grains slightly increases the magnitude $(<20 \mathrm{ppm})$ of Ba isotope variability in bulk meteorite samples that have high presolar grain concentrations. This conclusion could also apply to other isotope systems (e.g., Ti, $\mathrm{Cr}, \mathrm{Ni}$ ) and similar tests should be performed on comparable samples to test for the presence of nucleosynthetic anomalies and their dependence on dissolution protocol. The slight reduction in $\mathrm{Ba}$ isotope variation seen in the present dataset when compared to published data could also be partly explained by sampling bias. Evidence for this comes from the variations in the size of the ${ }^{135} \mathrm{Ba}$ isotope anomalies identified in fused samples from the same parent body and also from different pieces of the same meteorite on the $<0.8 \mathrm{~g}$ sampling scale. The cause of this variation in the ${ }^{135} \mathrm{Ba}$ anomaly likely results from the heterogeneous distribution of the presolar component (e.g., $\mathrm{SiC})$ on the sample scale of this study $(<0.8 \mathrm{~g})$ and potentially from a small contribution of radiogenic ${ }^{135} \mathrm{Ba}$. When looking closely, this variation in ${ }^{135} \mathrm{Ba}$ is also evident in published datasets where the samples have not undergone fusion before digestion. Future work to further constrain the cause(s) of ${ }^{135} \mathrm{Ba}$ variability in whole rock samples would lie in measurement of $\mathrm{Ba}$ isotope compositions in larger fused samples $(>0.8 \mathrm{~g})$ coupled with $\mathrm{Cs} / \mathrm{Ba}$ measurements on the same aliquots.

The origin of the nucleosynthetic isotope anomalies in ${ }^{135} \mathrm{Ba}$ and ${ }^{137} \mathrm{Ba}$ is likely a result of an $s$ process deficit in the feeding zones of the respective parent bodies. Regions with Ba isotope homogeneity in the early Solar System were present as evidenced by the $\mathrm{Ba}$ isotope compositions of H-chondrites, enstatite chondrites, eucrites, and Martian meteorites, which are all indistinguishable from terrestrial values. The origin of these isotopically homogeneous regions may represent a larger and, therefore, better mixed feeding zone. The preservation of only small $\mathrm{Ba}$ isotope anomalies on the bulk sample scale suggests that the highly anomalous $\mathrm{Ba}$ isotope presolar grain carriers were fairly well mixed in the solar nebula as compared to the Ti, $\mathrm{Cr}$, Mo, and $\mathrm{Ru}$ isotope carriers. This indicates that $\mathrm{Ba}$ isotope carriers were decoupled from those of $\mathrm{Ti}, \mathrm{Cr}, \mathrm{Mo}$, and $\mathrm{Ru}$. Perhaps the $\mathrm{Ba}$ isotope carriers either escaped destruction during nebular processing or they were emplaced into the solar nebula-forming region before the $\mathrm{Ti}, \mathrm{Cr}$, Mo, and Ru isotope carriers thus had a longer time to homogenize. 


\section{ACKNOWLEDGMENTS}

The authors would like to thank the Natural History Museum (UK), Smithsonian Natural History Museum (USA) and A. Bischoff the at the Institut für Planetologie (WWU) for providing samples and expertise during selection. Comments from L. Qin, R. Andreasen, an anonymous reviewer, and Associate Editor Yuri Amelin are gratefully acknowledged. Richard Walker is also thanked for constructive comments during preparation of the manuscript. Ursula Heitmann is thanked for her help during sample preparation, as are Tim Mock (DTM), Heidi Baier and Frank Deipenwisch (WWU) for their ongoing assistance in maintaining the laboratories and instruments. This work was funded by the European Commission through the Marie Curie Actions Research Training Networks ORIGINS project (ID: 35519), the Deutsche Forschungsgemeinschaft (Leibniz Prize to KM), the Helmholtz Gemeinschaft (HA-203 Planetary Evolution and Life to KM, EES), and NASA Cosmochemistry grant \#NNX10AG94G, all of which are gratefully acknowledged. 


\section{REFERENCES}

Ahrens L. H., Willis J. P. and Erlank A. J. (1973) The chemical composition of Kainsaz and Efremovka. Meteoritics 8, 133-139.

Anders E., Higuchi H., Ganapathy R. and Morgan J. W. (1976) Chemical fractionations in meteorites-IX. C3 chondrites. Geochim. Cosmochim. Acta 40, 1131-1139.

Anders, E. and Grevesse N. (1989) Abundances of the elements: Meteoritic and Solar. Geochim. Cosmochim. Acta 53, 197-214.

Andreasen R. and Sharma M. (2007) Mixing and homogenization in the early Solar System: Clues from Sr, Ba, Nd, and Sm isotopes in meteorites. Ap. J. 665, 874-883.

Andreasen R. and Sharma M. (2009) Fractionation and mixing in a thermal ionization mass spectrometer source: Implications and limitations for high precision $\mathrm{Nd}$ isotope analyses. Int. J. Mass Spec. 285, 49-57.

Arlandini C., Kappeler F., Wisshak K., Gallino R., Lugaro M., Busso M. and Straniero O. (1999) Neutron capture in low-mass asymptotic giant branch stars: cross sections and abundance signatures. Ap. J. 525, 886-900.

Arnold J.R. (1961) Nuclear effects of cosmic rays in meteorites. Ann. Rev. Nuclear. Sci. 11, 349.

Ávila J.N., Ireland T.R., Gyngard F., Zinner E., Mallmann G., Lugaro M., Holden P. and Amari S. (2013) Ba isotopic compositions in stardust $\mathrm{SiC}$ grains from the Murchison meteorite: Insights into the stellar origins of large $\mathrm{SiC}$ grains. Geochim. Cosmochim. Acta 120, 628647.

Barrat J.A., Zanda B., Moynier F., Bollinger C., Liorzou C., Bayo G. (2012) Geochemistry of CI chondrites: Major and trace elements, and $\mathrm{Cu}$ and $\mathrm{Zn}$ isotopes. Geochim. Cosmochim. Acta, 83, 79-92.

Becker R., Koller P., Morschl P., Kiesl W. and Hermann F. (1987) Determination of trace elements by neutron activation analysis in the Allende meteorite reference sample. In: Jarosewich E., Clarke R. S. Jr. and Barrows J. N., eds. The Allende Meteorite Reference Sample. Smithson. Contrib. Earth Sci., no. 27, 16-17. 
Bermingham K. R., Mezger K., Desch S., Scherer E.E. and Horstmann M. (2014) Evidence for extinct ${ }^{135} \mathrm{Cs}$ from Ba isotopes in Allende CAIs? Geochim. Cosmochim. Acta 133, 463478.

Bischoff A., Palme H., Ash A.D., Clayton R.N., Schultz L., Herpers U., Stöffler D., Grady M.M., Pillinger C.T., Spettel B., Weber H., Grund T., Endress M., Weber D. (1993) Paired Renazzo-type (CR) carbonaceous chondrites from the Sahara. Geochim. Cosmochim. Acta 57, 1587-1603.

Bischoff A., Gerel O., Buchwald V.F., Spettel B., Schultz L., Weber H.W., Schlüter J., Baljunnyam L., Borchuluun D., Cyambaa C., Garamjav D., Meteorites from Mongolia, Meteoritics \& Planet. Sci. 31, 152-157.

Brennecka G.A., Borg L.E. and Wadhwa M. (2013) Evidence for supernova injection into the solar nebula and the decoupling of $r$-process nucleosynthesis. Proc. Nat. Acad. Sci. 110, 17241-17246.

Burbidge E.M., Burbidge G.R., Fowler W.A. and Hoyle F. (1957) Synthesis of the elements in stars. Rev. Mod. Phys. 29, 547-650.

Burkhardt C., Kleine T., Oberli F., Pack A., Bourdon B. and Wieler R. (2011) Molybdenum isotope anomalies in meteorites: Constraints on solar nebula evolution and origin of the Earth. Earth Planet. Sci. Lett. 312, 390-400.

Carlson R.W., Boyet M. and Horan M. (2007) Chondrite barium, neodymium, and samarium isotopic heterogeneity and early Earth differentiation. Science 316, 1175-1178.

Day J.M., Taylor L.A., Floss C. and McSween Jr. H.Y. (2006). Petrology and chemistry of MIL 03346 and its significance in understanding the petrogenesis of nakhlites on Mars. Meteoritics \& Planet. Sci. 41, 581-606.

De Bièvre P. and Taylor P.D.P. (1993) IUPAC recommended isotopic abundances. Int. J. Mass Spectrom. Ion Phys. 123, 149.

Eugster O., Tera F. and Wasserburg G.J. (1969) Isotopic analyses of barium in meteorites and in terrestrial samples. J. Geophys. Res. 74, 3897-3908. 
Eugster O., Busemann H., Lorenzetti S., Terribilini D. (2002) Ejection ages from krypton-81krypton-83 dating and pre-atmospheric sizes and Martian meteorites. Meteoritics \& Planet. Sci. 37, 1345-1360.

Fujii T., Moynier F. and Albarède F. (2009). The nuclear field shift effect in chemical exchange reactions. Chem. Geol. 267, 139-156.

Fischer-Gödde M, Burkardt C., Kruijer T., Kleine T. (2015) Ru isotope heterogeneity in the solar protoplanetary disk. Geochim. Cosmochim. Acta 168, 151-171.

Fujiya W., Sugiura N., Hotta H., Ichimura K. and Sano Y. (2012) Evidence for the late formation of hydrous asteroids from young meteoritic carbonates. Nature Comm. 3, 627-633.

Fujiya W., Sugiura N., Sano Y. and Hiyagon H. (2013) Mn-Cr ages of dolomites in CI chondrites and the Tagish Lake ungrouped carbonaceous chondrite. Earth and Planet. Sci. 362, 130-142.

Grégoire M., Moine B.N., O'Reilly S.Y., Cottin J.Y. and Giret A. (2000) Trace element residence and partitioning in mantle xenoliths metasomatized by highly alkaline, silicateand carbonate-rich melts (Kerguelen Islands, Indian Ocean). J. Pet. 41, 477-509.

Harper H., Wiesmann H., Nyquist L.E. (1991) ${ }^{135} \mathrm{Cs}^{-}{ }^{135} \mathrm{Ba}$ : A new cosmochronometric constraint on the origin of the Earth and the astrophysical site of the origin of the Solar System. In Lunar and Planetary Inst., Abstracts for the 54th Annual Meeting of the Meteoritical Society. p 81.

Herzog G.F., Vogt S., Albrecht A., Xue S., Fink D., Klein J., Middleton R., Weber H.W., Schultz L., Complex exposure histories for meteorites with "short' exposure ages. Meteoritics \& Planet. Sci. 32, 413-422.

Heymann D. and Anders E. (1967) Meteorites with short cosmic-ray exposure ages, as determined from the ${ }^{26} \mathrm{Al}$ content. Geochim. Cosmochim. Acta 31, 1793-1809.

Hidaka H., Ohta Y., Yoneda S. and De Laeter J.R. (2001) Isotopic search for live ${ }^{135} \mathrm{Cs}$ in the early Solar System and possibility of ${ }^{135} \mathrm{Cs}^{-135} \mathrm{Ba}$ chronometer. Earth Planet. Sci. Lett. 193, 459-466. 
Hidaka K., Ohta Y. and Yoneda S. (2003) Nucleosynthetic components of the early Solar System inferred from Ba isotopic compositions in carbonaceous chondrites. Earth Planet. Sci. Lett. 214, 455-466.

Hidaka H. and Yoneda S. (2011) Neutron capture records of mesosiderites and an iron meteorite. Geochim. Cosmochim. Acta 75, 5706-5715.

Hidaka H. and Yoneda S. (2013) Radioactive Cs capture in the early Solar System. Sci. Rep. 3, 1330.

Huss G.R. and Lewis R.S. (1995) Presolar diamond, SiC, and graphite in primitive chondrites: Abundances as a function of meteorite class and petrologic type. Geochim. Cosmochim. Acta 59, 115-160.

Huss G.R., Meshik A.P., Smith J.B., Hohenberg C.M. (2003) Presolar diamond, silicon carbide, and graphite in carbonaceous chondrites: Implications for thermal processing in the solar nebula. Geochim. Cosmochim. Acta 67, 4823-4848.

Huss G.R., Ott U., Koscheev A.P. (2008) Noble gases in presolar diamonds III: Implications of ion implantation experiment with synthetic nanodiamonds. Meteoritics \& Planet. Sci. 43, 1811-1826.

Inghram M.G., Hayden R.J. and Hess Jr. D.C. (1947) The isotopic constitution of lanthanum and cerium. Phys. Rev. 72, 349-355.

Jilly C.E., Huss G.R., Krot A.N. and Nagashima K., Yin Q-Z. and Sugiura N. (2014) ${ }^{53} \mathrm{Mn}-{ }^{53} \mathrm{Cr}$ dating of aqueously formed carbonates in the CM2 lithology of the Sutter's Mill carbonaceous chondrite. Meteoritics \& Planet. Sci. 49, 2104-2117.

Keesom W.H. and van Dijk H. (1931) Possibility of separating neon into it isotopic components by distillation. Proc. Acad. Sci. Amsterdam 34, 42-50.

Knab H.-J. (1981) The distribution of trace elements in carbonaceous chondrites. Geochim. Cosmochim. Acta 45, 1563-1572. 
Krähenbühl U., Morgan J. W., Ganapathy R. and Anders E. (1973) Abundance of 17 trace elements in carbonaceous chondrites. Geochim. Cosmochim. Acta 37, 1131-1139.

Lee M.R. and Bland P.A. (2004) Mechanisms of weathering of meteorites recovered from hot and cold deserts and the formation of phyllosilicates. Geochim. Cosmochim. Acta $\mathbf{6 8}$, 893-916.

Lodders K. (2003) Solar System abundances and condensation temperatures of the elements. Ap. J. 591, 1220-1247.

Makide K., Nahashima K., Krot A.N., Huss G.R., Ciesla F.J., Hellebrand E., Gaidos E. and Yang L. (2011) Heterogeneous distribution of ${ }^{26} \mathrm{Al}$ at the birth of the Solar System, Ap. J. 733, L31-L35.

Marhas K.K., Hoppe P. and Ott U. (2007) NanoSIMS studies of Ba isotopic compositions in single presolar silicon carbide grains from AGB stars and supernovae. Meteoritics \& Planet. Sci. 42, 1077-1101.

Marti K and Graf T. (1992) Cosmic-ray exposure history of oridinary chondrites. Annu. Rev. Earth Planet. Sci. 20, 221-243.

Mazor E., Heymann D., Anders E. (1998) Nobel gases in carbonaceous chondrites. Geochim. Cosmochim. Acta 34, 781-824.

Miura Y.N, Nagao K., Sugiura N, Fujitani T., Warren P. (1998)Nobel gases, 81Kr-Kr exposure ages and 244Pu-Xe ages of six eucrites, Béréba, Binda, Camel Donga, Juvinas, Millbillillie, and Stannern. Geochim. Cosmochim. Acta 62, 2369-2387.

Morlok A., Bischoff A., Stephan T., Floss C., Zinner E. and Jessberger E.K. (2006) Brecciation and chemical heterogeneities of CI chondrites. Geochim. Cosmochim. Acta 70, 53715394.

Mughabghab S.F. (2003) Thermal neutron capture cross sections resonance integrals and Gfactors. International Nuclear Data Committee, 440. 
Nakai S., Shimizu H., Masuda A. (1986) A new geochronometer using lanthanum-138, Nature, 320, $433-435$.

Nichols, R.H., Brannon J.C., Podosek F.A. (2002) Excess 135-barium from live 135cesium in prgueil chemical separates. $33^{\text {rd }}$ Lunar and Planet. Sci. Conf. (Ab\#) 1929.

Pack A., Kremer K., Albrecht N., Simon K. and Kronz A. (2010) Description of an aerodynamic levitation apparatus with applications in Earth sciences. Geochem. Trans. 11, 4-16.

Pellin M.J., Calaway W.F., Davis A.M., Lewis R.S., Amari S., Clatyon R.N. (2000) Toward complete isotopic analysis of individual presolar silicon carbide grains: $\mathrm{C}, \mathrm{N}, \mathrm{Si}, \mathrm{Sr}, \mathrm{Zr}$, Mo, and Ba in single grains of Type X. $31^{\text {st }}$ Lunar and Planet. Sci. Conf. (Ab\#) 1917.

Pepin R.O. (1966) Heavy rare gases in silicates from the Estherville mesosiderite. J. Geophys. Res. 71, 2815-2829.

Qin L., Alexander C.M.O’D., Carlson R.W., Horan M.F. and Yokoyama T. (2010) Contributors to chromium isotope variations of meteorites. Geochim. Cosmochim. Acta 74, 1122-1145.

Qin L., Carlson R.W., Alexander C.M.O’D (2011) Correlated nucleosynthetic isotopic variability in $\mathrm{Cr}, \mathrm{Sr}, \mathrm{Ba}, \mathrm{Sm}, \mathrm{Nd}$ and $\mathrm{Hf}$ in Murchison and QUE 97008. Geochim. Cosmochim. Acta 75, 7806-7828.

Ranen M.C. and Jacobsen S.B. (2006) Barium isotopes in chondritic meteorites: Implications for planetary reservoir models. Science 314, 809-812.

Reynolds J.H. (1960) Isotopic composition of xenon from enstatite chondrites. Z. Naturforsch. 15a, 1112-1114.

Reynolds J.H. (1967) Isotopic abundance anomalies in the Solar System. Ann. Rev. Nucl. Sci. 17, 253-316.

Rocholl A., and Jochum K.P. (1993) Th, U and other trace elements in carbonaceous chondrites: Implications for the terrestrial and solar-system Th/U ratios. Earth Planet. Sci. 117, 265278. 
Roth A.S.G., Baur H., Heber V.S., Reusser E., Wieler R. (2011) Cosmogenic helium and neon in individual chondrules from Allende and Murchison: Implications for the precompaction exposure history of chondrules. Meteoritics \& Planet. Sci. 46, 989-1006.

Roth A.S.G., Scherer E.E., Maden C., Mezger K. and Bourdon B. (2014) Revisiting the ${ }^{142} \mathrm{Nd}$ deficits in the $1.48 \mathrm{Ga}$ Khariar alkaline rocks, India. Chem. Geol. 386, 238-248.

Rowe M.W. and Kuroda P.K. (1965) Fissiogenic xenon from the Pasamonte meteorite. J. Geophys. Res. 70, 709-714.

Rubin A.E., Warren P.H., Greenwood J.P., Verish R.S., Leshin L.A., Hervig R.L., Clayton R.N. and Mayeda T.K. (2000) Los Angeles: The most differentiated basaltic Martian meteorite. Geology 28, 1011-1014.

Savina M.R., Davis A.M., Tripa C.E., Pellin M.J., Clayton R.N., Lewis R.S., Amari C., Gallino, R. and Lugaro M. (2003) Barium isotopes in individual presolar silicon carbide grains from the Murchison meteorite. Geochim. Cosmochim. Acta 67, 3201-3214.

Schultz L. and Weber H.W. (1996) Nobel gases and H chondrite meteoid streams: No confirmation. J. Geophys. Res. 101, 21177-21181.

Srinivasan G., Ali A., Jabeen I. and Srinivasan S. (2011) Ba isotope composition of Tagish Lake meteorite. Lunar Planet. Sci. XLII, Lunar Planet. Inst., Houston. \#1953(abstr.).

Stracke A., Palme H., Gellissen M., Münker C., Kleine T., Birbaum K., Günther D., Bourdon B. and Zipfel J. (2012) Refractory element fractionation in the Allende meteorite: Implications for solar nebula condensation and the chondritic composition of planetary bodies. Geochim. Cosmochim. Acta 85, 114-141.

Terribilini D., Eugster O., Mittlefehldt D. W., Diamond L. W., Vogt S., and Wang D. (2000) Mineralogical and chemical composition and cosmic-ray exposure history of two mesosiderites and two iron meteorites. Meteoritics \& Planet. Sci., 35, 617-628.

Thiemens M.H. and Heidenreich III J.E. (1983) The mass-independent fractionation of oxygen: A novel isotope effect and its possible cosmochemical implications. Science 219, 10731075. 
Trinquier A., Elliott T., Ulfbeck D., Coath C., Krot A. and Bizzarro M. (2009) Origin of isotope heterogeneity in the solar protoplanetary disk. Science 324, 374-376.

Upadhyay D., Scherer E.E. and Mezger K. (2008) Fractionation and mixing of Nd isotopes during thermal ionization mass spectrometry: Implications for high precision ${ }^{142} \mathrm{Nd} /{ }^{144} \mathrm{Nd}$ analyses. J. Anal. At. Spectrom. 23, 561-568.

Urey H.C., Brickwedde F.G., and Murphy G.M. (1932a) A hydrogen isotope of mass 2 and its concentration. Phys. Rev. 40, 1-15.

Urey H.C., Brickwedde F.G., Murphy G.M. (1932b) A hydrogen isotope of mass 2. Phys. Rev. 39, 164-165.

Wittig N., Humayun H., Brandon A.D., Huang S. and Leya I. (2013) Coupled W-Os-Pt isotope systematics in IVB iron meteorites: In situ neutron dosimetry for W isotope chronology. Earth and Planet. Sci. 361, 152-161.

Wolf R., Richter G. R., Woodrow A. B. and Anders E. (1980) Chemical fractionations in meteorites--XI. C2 chondrites. Geochim. Cosmochim. Acta 44, 711-717.

Zinner E. (1998) Stellar nucleosynthesis and the isotopic composition of presolar grains from primitive meteorites. Ann. Rev. Earth. Planet. Sci. 26, 147-188. 
Table 1.

Natural abundances, nucleosynthetic origin, and neutron capture cross sections for Ba isotopes and ${ }^{135} \mathrm{Cs}$.

\begin{tabular}{cccc}
\hline Isotope & Abundance $(\%)^{*}$ & Nucleosynthetic origin $^{\dagger}$ & Neutron cross section $^{\wedge}$ (barns) \\
\hline${ }^{130} \mathrm{Ba}$ & 0.106 & $\mathrm{p}(100)$ & $8.7 \pm 0.9$ \\
${ }^{132} \mathrm{Ba}$ & 0.101 & $\mathrm{p}(100)$ & $6.5 \pm 0.8$ \\
${ }^{134} \mathrm{Ba}$ & 2.417 & $\mathrm{~s}(100)$ & $1.5 \pm 0.3$ \\
${ }^{135} \mathrm{Ba}$ & 6.592 & $\mathrm{~s}(26), \mathrm{r}(74)$ & $5.8 \pm 0.9$ \\
${ }^{136} \mathrm{Ba}$ & 7.854 & $\mathrm{~s}(100)$ & $0.68 \pm 0.2$ \\
${ }^{137} \mathrm{Ba}$ & 11.23 & $\mathrm{~s}(64), \mathrm{r}(36)$ & $3.6 \pm 0.2$ \\
${ }^{138} \mathrm{Ba}$ & 71.70 & $\mathrm{~s}(87), \mathrm{r}(13)$ & $0.40 \pm 0.04$ \\
${ }^{135} \mathrm{Cs}$ & - & $\mathrm{s}, \mathrm{r}$ & - \\
\hline
\end{tabular}

For each isotope, the main nucleosynthetic process and the percentage proportion that it represents are shown in parentheses (except for ${ }^{135} \mathrm{Cs}$ ).

* Present day natural isotope abundances (De Bièvre and Taylor, 1993)

${ }^{\dagger}$ Nucleosynthetic proportions of Ba isotopes (Arlandini et al., 1999)

${ }^{\wedge}$ Thermal neutron capture cross sections (Mughabghab, 2003) 
Table 2. List of studied meteorites.

\begin{tabular}{|c|c|c|c|c|c|c|}
\hline Sample & Type & $\mathrm{Ba}(\mathrm{ppm})$ & $\begin{array}{c}\text { Mass } \\
(\mathrm{g})\end{array}$ & Laser fusion & Laboratory & $\begin{array}{c}\text { Cosmic ray exposure } \\
\text { age }(\mathrm{Ma})\end{array}$ \\
\hline $\begin{array}{l}\text { Terrestrial basalt } \\
\text { DU17 }\end{array}$ & Basalt & 33 & 0.104 & $\mathrm{~N}$ & WWU & N/A \\
\hline \multicolumn{7}{|l|}{$H E D$} \\
\hline Juvinas & EUC & 30 & 0.072 & $\mathrm{~N}$ & WWU & 11 \\
\hline Stannern $_{\text {fine }} \S$ & EUC & 53 & 0.097 & $\mathrm{~N}$ & WWU & 35 \\
\hline Stannern $_{\text {coarse }} \S$ & EUC & 53 & 0.099 & $\mathrm{~N}$ & WWU & 35 \\
\hline \multicolumn{7}{|l|}{$\begin{array}{l}\text { Ordinary } \\
\text { chondrites }\end{array}$} \\
\hline Oesede & H5 & - & 0.504 & $\mathrm{~N}$ & WWU & 41 \\
\hline Tugalin-Bulen & H6 & - & 0.456 & $\mathrm{~N}$ & WWU & 20 \\
\hline Butsura & H6 & 1.1 & 0.537 & $\mathrm{~N}$ & WWU & 41 \\
\hline Guenie-Gao & $\mathrm{H} 4 / 5$ & - & 0.475 & $\mathrm{Y}$ & WWU & 10 \\
\hline \multicolumn{7}{|l|}{$\begin{array}{l}\text { Enstatite } \\
\text { chondrites }\end{array}$} \\
\hline Indarch & EH4 & 2.3 & 0.443 & $\mathrm{Y}$ & WWU & $6.5-16$ \\
\hline \multicolumn{7}{|l|}{$\begin{array}{l}\text { Carbonaceous } \\
\text { chondrites }\end{array}$} \\
\hline Orgueil 1 & CI & 2.3 & 0.738 & $\mathrm{Y}$ & WWU & 4.5 \\
\hline Orgueil 2 & $\mathrm{CI}$ & 2.3 & 0.821 & Y & DTM & 4.5 \\
\hline Renazzo $^{\#}$ & CR2 & 3.4 & 0.844 & $\mathrm{Y}$ & WWU & 9 \\
\hline Cold Bokkeveld & $\mathrm{CM} 2$ & - & 0.725 & $\mathrm{Y}$ & DTM & 0.3 \\
\hline Mighei & CM2 & 2.5 & 0.685 & $\mathrm{Y}$ & DTM & 4.0 \\
\hline Murchison & CM2 & 1.6 & 0.598 & $\mathrm{Y}$ & WWU & 4.3 \\
\hline Allende $_{\text {acid }}$ & CV3(ox) & 5.3 & 0.516 & $\mathrm{~N}$ & DTM & $1.6-5.5$ \\
\hline Allende $_{\text {fused }}$ & CV3(ox) & 5.3 & 0.610 & $\mathrm{Y}$ & DTM & $1.6-5.5$ \\
\hline Vigarano & CV3 (red) & 5.2 & 0.482 & $\mathrm{Y}$ & DTM & - \\
\hline Lancé & $\mathrm{CO} 3.5$ & - & 0.473 & $\mathrm{Y}$ & DTM & 3.6 \\
\hline Kainsaz & $\mathrm{CO} 3.2$ & 3.9 & 0.946 & $\mathrm{Y}$ & DTM & 20.9 \\
\hline \multicolumn{7}{|l|}{$S N C$} \\
\hline Zagami & Shergottite & 26 & 0.067 & $\mathrm{~N}$ & WWU & 2.9 \\
\hline $\begin{array}{l}\text { Shergotty } \\
\text { (USNM 6676) }\end{array}$ & Shergottite & 34 & 0.053 & $\mathrm{~N}$ & WWU & 3.0 \\
\hline$\underset{\text { (USNM 7058) }}{\text { Los Angeles } 001^{\dagger}}$ & Shergottite & 47 & 0.076 & $\mathrm{~N}$ & WWU & 3.1 \\
\hline NWA $4864^{\dagger}$ & Shergottite & 26 & 0.070 & $\mathrm{~N}$ & WWU & - \\
\hline Nakhla & Nakhlite & 32 & 0.055 & $\mathrm{~N}$ & WWU & 11 \\
\hline $\begin{array}{l}\text { MIL 03346 } \\
\text { subsample 193) }\end{array}$ & Nakhlite & 26 & 0.072 & $\mathrm{~N}$ & WWU & 9.5 \\
\hline
\end{tabular}

Type of meteorite, bulk sample Ba estimated from previous publications, mass of sample analyzed, indication of analytical treatment, laboratory where samples were processed, and sample repository location are listed. All samples are falls except for those marked $\left({ }^{\dagger}\right)$.

${ }^{\S}$ Fine or coarse-grained texture evident within whole rock chip

\# Mineral separates (non-metal fraction) 
Table 3. The Ba isotope data for terrestrial basalt (DU17), eucrites, and ordinary chondrites analyzed in the present study.

\begin{tabular}{|c|c|c|c|c|}
\hline & ${ }^{132} \mathrm{Ba} /{ }^{138} \mathrm{Ba}$ & ${ }^{135} \mathrm{Ba} /{ }^{138} \mathrm{Ba}$ & ${ }^{136} \mathrm{Ba} /{ }^{138} \mathrm{Ba}$ & ${ }^{137} \mathrm{Ba} /{ }^{138} \mathrm{Ba}$ \\
\hline \multicolumn{5}{|l|}{ Ba standard } \\
\hline Ba std 01 & 0.00141295 & $0.0919379(3)$ & $0.1095349(3)$ & 0.1565298 \\
\hline Ba std 02 & 0.00141325 & $0.0919386(3)$ & $0.1095352(2)$ & $0.1565306(2)$ \\
\hline Ba std 03 & 0.00141319 (4) & $0.0919377(3)$ & 0.1095348 & $0.1565297(2)$ \\
\hline Ba std 04 & 0.00141338 & $0.0919376(2)$ & $0.1095325(2)$ & $0.1565287(2)$ \\
\hline Ba std 05 & 0.00141343 & $0.0919377(2)$ & $0.1095335(2)$ & $0.1565296(2)$ \\
\hline Ba std 06 & 0.00141334 & $0.0919375(3)$ & $0.1095356(2)$ & $0.1565289(2)$ \\
\hline Ba std 07 & 0.00141345 & 0.0919386 & $0.1095342(2)$ & $0.1565303(2)$ \\
\hline Ba std 08 & 0.00141333 (4) & $0.0919378(2)$ & $0.1095326(2)$ & $0.1565304(2)$ \\
\hline Ba std 09 & $0.00141347(3)$ & $0.0919368(2)$ & $0.1095332(2)$ & $0.1565313(2)$ \\
\hline Average & 0.00141331 & 0.0919378 & 0.1095340 & 0.1565299 \\
\hline External precision $(2 \sigma, \mathrm{ppm})$ & 229 & 12 & 21 & 11 \\
\hline DU17A 01 & 0.00141342 & $0.0919369(3)$ & $0.1095351(2)$ & $0.1565291(2)$ \\
\hline DU17A 02 & 0.00141328 & $0.0919374(3)$ & 0.1095311 & $0.1565302(2)$ \\
\hline DU17A 03 & $0.00141342(5)$ & 0.0919379 (3) & $0.1095346(2)$ & 0.1565298 \\
\hline DU17B 01 & 0.00141330 & $0.0919383(3)$ & $0.1095342(3)$ & 0.1565304 \\
\hline Average deviation from Ba std (ppm) & $31( \pm 107)$ & $-2( \pm 13)$ & $-1( \pm 25)$ & $0( \pm 7)$ \\
\hline Juvinas 01 & $0.00141328(4)$ & $0.0919382(3)$ & $0.1095356(2)$ & $0.1565306(3)$ \\
\hline Juvinas 02 & $0.00141333(4)$ & $0.0919380(3)$ & $0.1095351(2)$ & $0.1565306(2)$ \\
\hline Juvinas 03 & $0.00141350(3)$ & $0.0919381(2)$ & $0.1095360(2)$ & $0.1565305(2)$ \\
\hline Juvinas 04 & $0.00141320(4)$ & $0.0919384(3)$ & $0.1095350(2)$ & $0.1565308(2)$ \\
\hline Average deviation from Ba std ( $\mathrm{ppm}$ ) & $12( \pm 181)$ & $4( \pm 4)$ & $13( \pm 9)$ & $4( \pm 1)$ \\
\hline Stannern (C) 01 & $0.00141335(4)$ & $0.0919378(2)$ & $0.1095347(2)$ & $0.1565296(2)$ \\
\hline Stannern (C) 02 & $0.00141346(4)$ & $0.0919386(2)$ & $0.1095363(2)$ & $0.1565306(2)$ \\
\hline Stannern (C) 03 & $0.00141333(4)$ & $0.0919385(2)$ & $0.1095342(2)$ & $0.1565306(3)$ \\
\hline Stannern (C) 04 & $0.00141319(4)$ & $0.0919382(3)$ & $0.1095349(2)$ & $0.1565308(2)$ \\
\hline Average deviation from Ba std (ppm) & $47( \pm 155)$ & $5( \pm 8)$ & $9( \pm 17)$ & $3( \pm 7)$ \\
\hline Stannern $(F) 01$ & $0.00141334(4)$ & $0.0919378(3)$ & $0.1095343(2)$ & $0.1565299(2)$ \\
\hline Stannern $(F) 03$ & $0.00141333(4)$ & $0.0919378(2)$ & $0.1095348(2)$ & $0.1565302(2)$ \\
\hline Stannern $(\mathrm{F}) 04$ & $0.00141345(4)$ & $0.0919381(2)$ & $0.1095359(2)$ & $0.1565299(2)$ \\
\hline Stannern $(\mathrm{F}) 05$ & $0.00141338(3)$ & $0.0919381(2)$ & $0.1095341(2)$ & $0.1565306(2)$ \\
\hline Average deviation from $\mathrm{Ba}$ std ( $\mathrm{ppm}$ ) & $47( \pm 77)$ & $4( \pm 3)$ & $11( \pm 15)$ & $4( \pm 5)$ \\
\hline Butsura 01 & $0.00141360(4)$ & $0.0919373(2)$ & $0.1095360(3)$ & $0.1565307(2)$ \\
\hline Butsura 02 & $0.00141359(4)$ & $0.0919365(3)$ & $0.1095316(2)$ & $0.1565297(2)$ \\
\hline Average deviation from Ba std (ppm) & $200( \pm 7)$ & $-10( \pm 13)$ & $-2( \pm 56)$ & $2( \pm 8)$ \\
\hline Oesede 01 & $0.00141341(4)$ & $0.0919356(3)$ & $0.1095379(3)$ & $0.1565281(3)$ \\
\hline Oesede 02 & $0.00141339(5)$ & $0.0919365(3)$ & $0.1095369(3)$ & $0.1565283(3)$ \\
\hline Average deviation from Ba std (ppm) & $-37( \pm 18)$ & $-3( \pm 13)$ & $16( \pm 13)$ & $-3( \pm 2)$ \\
\hline Tugalin-Bulen 01 & $0.0014135(5)$ & $0.0919383(3)$ & $0.1095330(3)$ & $0.1565314(3)$ \\
\hline Tugalin-Bulen 02 & $0.0014134(4)$ & $0.0919367(3)$ & $0.1095361(3)$ & $0.1565287(3)$ \\
\hline Average deviation from $\mathrm{Ba}$ std (ppm) & $117( \pm 126)$ & $-3( \pm 24)$ & $4( \pm 41)$ & $1( \pm 25)$ \\
\hline $\begin{array}{l}\text { Barium data are normalized to }{ }^{134} \mathrm{Ba} /{ }^{1} \\
\text { in the last digits in parentheses are } 2 \\
\text { standard measurements. Number of ir } \\
\text { the Ba standard values are } 2 \sigma \text { of the } \\
\text { one analysis was performed, the value } \\
\text { replicate standards analyzed (in ppm) }\end{array}$ & $\begin{array}{l}03371 \text { (De Bièvre } \\
\text { egrations within } \\
\text { s per analysis (n) } \\
\text { amples analyses } \\
\text { theses after the a }\end{array}$ & $\begin{array}{l}\text { Taylor, 1993; } \\
\text { halysis. The ex } \\
\text { 20. Values (in } \\
\text { pm where mor } \\
\text { ge deviation fro }\end{array}$ & $\begin{array}{l}\text { precision is b } \\
\text { heses) after th } \\
\text { one analysis } \\
\text { Ba standard }\end{array}$ & $\begin{array}{l}007) \text {. Uncertaint } \\
\text { n } 2 \sigma \text { of } 9 \text { replic } \\
\text { age deviation fro } \\
\text { sssible. When or } \\
\text { represent } 2 \sigma \text { of }\end{array}$ \\
\hline
\end{tabular}


Table 4.

The $\mathrm{Ba}$ isotope data from Martian meteorites analyzed in the present study.

\begin{tabular}{|c|c|c|c|c|}
\hline & ${ }^{132} \mathrm{Ba} /{ }^{138} \mathrm{Ba}$ & ${ }^{135} \mathrm{Ba} /{ }^{138} \mathrm{Ba}$ & ${ }^{136} \mathrm{Ba} /{ }^{138} \mathrm{Ba}$ & ${ }^{137} \mathrm{Ba} /{ }^{138} \mathrm{Ba}$ \\
\hline \multicolumn{5}{|l|}{ Ba standard } \\
\hline Ba std 01 & $0.00141347(4)$ & $0.0919372(2)$ & $0.1095353(2)$ & $0.1565289(2)$ \\
\hline Ba std 02 & $0.00141354(4)$ & $0.0919377(2)$ & $0.1095354(2)$ & $0.1565282(2)$ \\
\hline Ba std 03 & $0.00141353(4)$ & $0.0919370(2)$ & $0.1095348(2)$ & $0.1565267(2)$ \\
\hline Ba std 04 & $0.00141357(4)$ & $0.0919383(2)$ & $0.1095350(2)$ & $0.1565287(2)$ \\
\hline Ba std 05 & $0.00141346(4)$ & $0.0919367(2)$ & $0.1095333(2)$ & $0.1565264(2)$ \\
\hline Ba std 06 & $0.00141328(4)$ & $0.0919379(3)$ & $0.1095355(2)$ & $0.1565269(2)$ \\
\hline Average & 0.00141348 & 0.0919375 & 0.1095349 & 0.1565277 \\
\hline External precision $(2 \sigma, \mathrm{ppm})$ & 149 & 13 & 15 & 14 \\
\hline Los Angeles 001 & $0.00141347(4)$ & $0.0919369(3)$ & $0.1095363(2)$ & $0.1565279(2)$ \\
\hline Average deviation from Ba std (ppm) & $-1( \pm 149)$ & $-7( \pm 13)$ & $13( \pm 15)$ & $1( \pm 14)$ \\
\hline NWA4864 & $0.00141360(4)$ & $0.0919381(3)$ & $0.1095338(2)$ & $0.1565285(3)$ \\
\hline Average deviation from Ba std (ppm) & $89( \pm 149)$ & $7( \pm 13)$ & $-10( \pm 15)$ & $6( \pm 14)$ \\
\hline MIL03346 & $0.00141343(6)$ & $0.0919385(3)$ & $0.1095347(3)$ & $0.1565297(3)$ \\
\hline Average deviation from Ba std (ppm) & $-29( \pm 149)$ & $12( \pm 13)$ & $-1( \pm 15)$ & $13( \pm 14)$ \\
\hline Nakhla 01 & $0.00141361(4)$ & $0.0919367(2)$ & $0.1095375(2)$ & $0.1565276(2)$ \\
\hline Nakhla 02 & $0.00141336(4)$ & $0.0919365(2)$ & $0.1095364(2)$ & $0.1565264(2)$ \\
\hline Average deviation from Ba std (ppm) & $5( \pm 253)$ & $-10( \pm 4)$ & $19( \pm 14)$ & $-4( \pm 12)$ \\
\hline Zagami 01 & $0.00141370(4)$ & $0.0919376(3)$ & $0.1095368(2)$ & $0.1565288(2)$ \\
\hline Zagami 02 & $0.00141347(4)$ & $0.0919376(2)$ & $0.1095362(2)$ & $0.1565282(2)$ \\
\hline Average deviation from Ba std (ppm) & $80( \pm 232)$ & $2( \pm 1)$ & $15( \pm 8)$ & $5( \pm 6)$ \\
\hline Shergotty & $0.00141355(4)$ & $0.0919385(2)$ & $0.10953537(2)$ & $0.1565278(2)$ \\
\hline Average deviation from Ba std (ppm) & $55( \pm 149)$ & $11( \pm 13)$ & $4( \pm 15)$ & $1( \pm 14)$ \\
\hline
\end{tabular}

Barium data are normalized to ${ }^{134} \mathrm{Ba} /{ }^{138} \mathrm{Ba}=0.03371$ (De Bièvre and Taylor, 1993; Andreasen and Sharma, 2007). Uncertainties in the last digits in parentheses are 2 s.e. of integrations during an analysis. The external precision is based on $2 \sigma$ of 6 replicate standard measurements. Number of integrations per analysis (n) is 320. Values (in parentheses) after the average deviation from the $\mathrm{Ba}$ standard values are $2 \sigma$ of the replicate samples analyses in ppm or $2 \sigma$ of 6 replicate standard measurements (in ppm) when no replicate analyses were available. 
Table 5. The Ba isotope data from carbonaceous chondrites, enstatite chondrites, thermally unequilibrated meteorites analyzed in the present study.

\begin{tabular}{|c|c|c|c|c|}
\hline & ${ }^{132} \mathrm{Ba} /{ }^{138} \mathrm{Ba}$ & ${ }^{135} \mathrm{Ba} /{ }^{138} \mathrm{Ba}$ & ${ }^{136} \mathrm{Ba} /{ }^{138} \mathrm{Ba}$ & ${ }^{137} \mathrm{Ba} /{ }^{138} \mathrm{Ba}$ \\
\hline \multicolumn{5}{|l|}{ Ba standards } \\
\hline Ba std 01 & $0.00141353(4)$ & $0.0919370(2)$ & $0.1095348(2)$ & $0.1565267(2)$ \\
\hline Ba std 02 & $0.00141357(4)$ & $0.0919383(2)$ & $0.1095350(2)$ & $0.1565287(2)$ \\
\hline Ba std 03 & $0.00141337(4)$ & $0.0919382(2)$ & $0.1095346(2)$ & $0.1565278(2)$ \\
\hline Ba std 04 & $0.00141346(4)$ & $0.0919367(2)$ & $0.1095333(2)$ & $0.1565264(2)$ \\
\hline Ba std 05 & $0.00141338(4)$ & $0.0919372(2)$ & $0.1095373(2)$ & $0.1565272(2)$ \\
\hline Ba std 06 & $0.00141338(4)$ & $0.0919376(2)$ & $0.1095320(2)$ & $0.1565273(2)$ \\
\hline Ba std 07 & $0.00141335(3)$ & $0.0919379(2)$ & $0.1095357(2)$ & $0.1565259(2)$ \\
\hline Ba std 08 & $0.00141328(4)$ & $0.0919379(3)$ & $0.1095355(2)$ & $0.1565269(2)$ \\
\hline Average & 0.00141341 & 0.0919376 & 0.1095348 & 0.1565271 \\
\hline External precision $(2 \sigma, \mathrm{ppm})$ & 138 & 12 & 29 & 11 \\
\hline Renazzo 01 & $0.00141326(4)$ & $0.0919390(3)$ & $0.1095334(3)$ & $0.1565281(3)$ \\
\hline Renazzo $02(n=200)$ & $0.00141323(5)$ & $0.0919398(4)$ & $0.1095367(4)$ & $0.1565286(3)$ \\
\hline$A v \cdot$ dev. from Ba std (ppm) & $-120( \pm 22)$ & $20( \pm 12)$ & $2( \pm 43)$ & $8( \pm 4)$ \\
\hline Orgueil 1 & $0.00141357(4)$ & $0.0337039(2)$ & $0.0919425(2)$ & $0.1095327(2)$ \\
\hline$A v \cdot$ dev. from Ba std (ppm) & $109( \pm 138)$ & $53( \pm 12)$ & $-19( \pm 29)$ & $23( \pm 11)$ \\
\hline Gao-Guenie & $0.00141366(4)$ & $0.0919381(3)$ & $0.1095356(2)$ & $0.1565273(2)$ \\
\hline Av. dev. from Ba std (ppm) & $176( \pm 138)$ & $5( \pm 12)$ & $8( \pm 29)$ & $1( \pm 11)$ \\
\hline Murchison & $0.00141336(4)$ & $0.0919389(2)$ & $0.1095344(3)$ & $0.1565273(3)$ \\
\hline Av. dev. from Ba std (ppm) & $-39( \pm 138)$ & $14( \pm 12)$ & $-4( \pm 29)$ & $1( \pm 11)$ \\
\hline Indarch $(\mathrm{n}=170)$ & $0.00141328(9)$ & $0.0919385(4)$ & $0.1095309(5)$ & $0.1565292(4)$ \\
\hline Av. dev. from Ba std (ppm) & $-93( \pm 138)$ & $10( \pm 12)$ & $-35( \pm 29)$ & $13( \pm 11)$ \\
\hline
\end{tabular}

Barium data are normalized to ${ }^{134} \mathrm{Ba} /{ }^{138} \mathrm{Ba}=0.03371$ (De Bièvre and Taylor, 1993; Andreasen and Sharma, 2007). Uncertainties in the last digits (in parentheses) are 2 s.e. of integrations during an analysis. The external precision is based on $2 \sigma$ of 8 replicate standard measurements. Number of integrations per analysis (n) is 320. Values (in parentheses) after the average deviation from the Ba standard values are $2 \sigma$ of the replicate samples analyses in $\mathrm{ppm}$. 
Table 6. The Ba isotope data from carbonaceous chondrites analyzed in the present study.

\begin{tabular}{|c|c|c|c|c|c|}
\hline & ${ }^{130} \mathrm{Ba} /{ }^{138} \mathrm{Ba}$ & ${ }^{132} \mathrm{Ba} /{ }^{138} \mathrm{Ba}$ & ${ }^{135} \mathrm{Ba} /{ }^{138} \mathrm{Ba}$ & ${ }^{136} \mathrm{Ba} /{ }^{138} \mathrm{Ba}$ & ${ }^{137} \mathrm{Ba} /{ }^{138} \mathrm{Ba}$ \\
\hline \multicolumn{6}{|l|}{ Ba standard } \\
\hline Ba std 01 & $0.00147580(5)$ & $0.00141226(5)$ & $0.0919090(3)$ & $0.1095073(2)$ & 0.1565203 \\
\hline Ba std 02 & $0.00147556(3)$ & 0.00141228 & $0.0919091(2)$ & $0.1095072(2)$ & $0.1565193(2)$ \\
\hline Ba std 03 & $0.00147570(3)$ & $0.00141225(3)$ & $0.0919092(2)$ & $0.1095082(2)$ & $0.1565203(2)$ \\
\hline Ba std 04 & 0.00147578 & $0.00141227(3)$ & 0.0919097 (2) & $0.1095048(2)$ & $0.1565203(2)$ \\
\hline Ba std 05 & $0.00147575(3)$ & 0.00141228 & 0.0919097 (2) & $0.1095084(2)$ & $0.1565201(2)$ \\
\hline Ba std 06 & $0.00147569(3)$ & $0.00141231(3)$ & $0.0919094(2)$ & $0.1095069(2)$ & $0.1565211(2)$ \\
\hline Ba std 07 & $0.00147572(3)$ & $0.00141226(3)$ & $0.0919093(2)$ & 0.1095077 (2) & $0.1565210(2)$ \\
\hline Ba std 08 & 0.00147575 & $0.00141218(2)$ & $0.0919096(2)$ & $0.1095055(2)$ & $0.1565215(2)$ \\
\hline Ba std 09 & $0.00147576(3)$ & $0.00141227(3)$ & $0.0919093(2)$ & $0.1095060(2)$ & $0.1565211(2)$ \\
\hline Ba std 10 & $0.00147581(3)$ & $0.00141223(2)$ & $0.0919096(2)$ & 0.1095059 (2) & $0.1565206(2)$ \\
\hline Ba std 11 & $0.00147573(3)$ & $0.00141218(2)$ & $0.0919090(2)$ & 0.1095059 (2) & $0.1565203(2)$ \\
\hline Ba std 12 & 0.00147570 & $0.00141236(3)$ & 0.0919098 (3) & 0.1095059 (2) & $0.1565209(2)$ \\
\hline Ba std 13 & $0.00147585(2)$ & $0.00141229(2)$ & $0.0919088(2)$ & $0.1095042(1)$ & $0.1565200(1)$ \\
\hline Ba std 14 & $0.00147572(3)$ & $0.00141245(3)$ & $0.0919088(2)$ & $0.1095075(2)$ & $0.1565197(2)$ \\
\hline Ba std 15 & $0.00147564(3)$ & $0.00141219(3)$ & 0.0919089 (2) & $0.1095077(2)$ & $0.1565202(2)$ \\
\hline Ba std 16 & $0.00147583(3)$ & $0.00141230(3)$ & $0.0919094(2)$ & $0.1095083(2)$ & $0.1565205(2)$ \\
\hline Ba std 17 & $0.00147576(3)$ & $0.00141240(3)$ & $0.0919087(2)$ & $0.1095071(2)$ & $0.1565203(2)$ \\
\hline Ba std 18 & $0.00147572(2)$ & $0.00141212(2)$ & $0.0919095(2)$ & $0.1095059(2)$ & $0.1565211(2)$ \\
\hline Ba std 19 & $0.00147576(2)$ & $0.00141230(2)$ & $0.0919094(2)$ & $0.1095061(1)$ & 0.1565215 \\
\hline Ba std 20 & $0.00147571(2)$ & $0.00141224(2)$ & $0.0919092(2)$ & $0.1095057(2)$ & $0.1565206(2)$ \\
\hline Ba std 21 & $0.00147583(4)$ & $0.00141210(4)$ & $0.0919096(3)$ & $0.1095069(3)$ & $0.1565213(3)$ \\
\hline Average & 0.00147574 & 0.00141226 & 0.0919093 & 0.1095066 & 0.1565206 \\
\hline External precision $(2 \sigma, \mathrm{ppm})$ & 92 & 117 & 7 & 21 & 7 \\
\hline Allende_acid 01 & $0.00147578(3)$ & $0.00141228(3)$ & $0.0919126(2)$ & $0.1095089(2)$ & $0.1565235(2)$ \\
\hline Allende_acid 02 & $0.00147561(4)$ & $0.00141237(4)$ & $0.0919119(2)$ & $0.1095076(2)$ & $0.1565232(2)$ \\
\hline Av. dev. from Ba std (ppm) & $-33( \pm 160)$ & $41( \pm 89)$ & $32( \pm 11)$ & $15( \pm 17)$ & $18( \pm 2)$ \\
\hline Allende_fused 01 & $0.00147574(2)$ & $0.00141218(2)$ & $0.0919130(2)$ & $0.1095080(2)$ & $0.1565242(2)$ \\
\hline Allende_fused $02(n=280)$ & $0.00147554(3)$ & $0.00141219(3)$ & $0.0919123(2)$ & $0.1095074(2)$ & $0.1565233(2)$ \\
\hline Av. dev. from Ba std (ppm) & $-67( \pm 188)$ & $-54( \pm 13)$ & $37( \pm 11)$ & $10( \pm 7)$ & $20( \pm 8)$ \\
\hline Cold Bokkeveld 01 & $0.00147568(3)$ & $0.00141222(3)$ & $0.0919125(2)$ & $0.1095066(2)$ & $0.1565229(2)$ \\
\hline Cold Bokkeveld 02 & $0.00147580(3)$ & $0.00141224(2)$ & $0.0919122(2)$ & $0.1095067(2)$ & $0.1565228(2)$ \\
\hline Cold Bokkeveld 03 & $0.00147561(2)$ & $0.00141223(3)$ & $0.0919124(2)$ & $0.1095070(2)$ & $0.1565231(2)$ \\
\hline Av. dev. from Ba std (ppm) & $-29( \pm 125)$ & $-23( \pm 18)$ & $34( \pm 3)$ & $1( \pm 3)$ & $15( \pm 3)$ \\
\hline Mighei $(n=480)$ & $0.00147578(3)$ & $0.00141217(3)$ & $0.0919106(2)$ & $0.1095074(2)$ & $0.1565220(2)$ \\
\hline Av. dev. from Ba std (ppm) & $25( \pm 92)$ & $-65( \pm 117)$ & $14( \pm 7)$ & $7( \pm 21)$ & $9( \pm 7)$ \\
\hline Kainsaz 01 & $0.00147572(3)$ & $0.00141218(3)$ & $0.0919125(2)$ & $0.1095061(2)$ & $0.1565233(2)$ \\
\hline Kainsaz $02(n=330)$ & $0.00147568(5)$ & $0.00141230(4)$ & $0.0919124(4)$ & $0.1095052(4)$ & $0.1565227(4)$ \\
\hline Av. dev. from Ba std (ppm) & $-26( \pm 39)$ & $-16( \pm 125)$ & $34( \pm 1)$ & $-9( \pm 12)$ & $16( \pm 5)$ \\
\hline Lancé 01 & $0.00147574(3)$ & $0.00141225(3)$ & $0.0919125(2)$ & $0.1095054(2)$ & $0.1565231(2)$ \\
\hline Lancé 02 & $0.00147562(2)$ & $0.00141228(2)$ & $0.0919129(2)$ & $0.1095069(2)$ & $0.1565240(2)$ \\
\hline Av. dev. from Ba std (ppm) & $-44( \pm 118)$ & $3( \pm 29)$ & $37( \pm 7)$ & $-4( \pm 19)$ & $19( \pm 8)$ \\
\hline Vigarano 01 & $0.00147575(3)$ & $0.00141223(3)$ & $0.0919122(2)$ & $0.1095047(2)$ & $0.1565231(2)$ \\
\hline Vigarano 02 & $0.00147589(2)$ & $0.00141220(2)$ & $0.0919126(2)$ & $0.1095047(2)$ & $0.1565232(2)$ \\
\hline Vigarano 03 & $0.00147563(3)$ & $0.00141211(3)$ & $0.0919134(2)$ & $0.1095068(2)$ & $0.1565243(2)$ \\
\hline Av. dev. from Ba std (ppm) & $10( \pm 178)$ & $-58( \pm 89)$ & $38( \pm 13)$ & $-11( \pm 23)$ & $19( \pm 9)$ \\
\hline Orgueil 01 & $0.00147566(3)$ & $0.00141223(3)$ & $0.0919113(2)$ & $0.1095068(2)$ & $0.1565228(2)$ \\
\hline Orgueil 02 & $0.00147535(3)$ & $0.00141217(3)$ & $0.0919113(2)$ & $0.1095077(2)$ & $0.1565234(2)$ \\
\hline Av. dev. from Ba std (ppm) & $-161( \pm 295)$ & $-44( \pm 67)$ & $22( \pm 1)$ & $6( \pm 12)$ & $16( \pm 5)$ \\
\hline
\end{tabular}

Barium data are normalized to ${ }^{134} \mathrm{Ba} /{ }^{138} \mathrm{Ba}=0.03371$ (De Bièvre and Taylor, 1993; Andreasen and Sharma, 2007). Uncertainties in the last digits (in parentheses) are 2 s.e. of integrations during an analysis. The external precision is based on $2 \sigma$ of 21 replicate 
standard measurements. Number of integrations per analysis (n) is 540. Values (in parentheses) after the average deviation from the Ba standard values are $2 \sigma$ of the replicate samples analyses in $\mathrm{ppm}$. 
Table 7. Comparison of Ba isotope compositions of different aliquots from the same meteorites from present study and Carlson et al. (2007). Values (in parentheses) are $2 \sigma$ of the replicate standard analyses in ppm for that analytical session. All data normalized using ${ }^{134} \mathrm{Ba} /{ }^{138} \mathrm{Ba}=0.03371$ (De Bièvre and Taylor, 1993; Andreasen and Sharma, 2007).

\begin{tabular}{|c|c|c|c|c|c|c|}
\hline & ${ }^{130} \mathrm{Ba} /{ }^{138} \mathrm{Ba}$ & ${ }^{132} \mathrm{Ba} /{ }^{138} \mathrm{Ba}$ & ${ }^{135} \mathrm{Ba} /{ }^{138} \mathrm{Ba}$ & ${ }^{136} \mathrm{Ba} /{ }^{138} \mathrm{Ba}$ & ${ }^{137} \mathrm{Ba} /{ }^{138} \mathrm{Ba}$ & Source \\
\hline \multicolumn{7}{|l|}{ CM2 } \\
\hline Cold Bokkeveld & $-29( \pm 92)$ & $-23( \pm 117)$ & $34( \pm 7)$ & $1( \pm 21)$ & $15( \pm 7)$ & WWU \\
\hline Cold Bokkeveld & $174( \pm 108)$ & $102( \pm 204)$ & $53( \pm 11)$ & $-6( \pm 12)$ & $23( \pm 12)$ & Carlson et al. (2007) \\
\hline Mighei & $25( \pm 92)$ & $-65( \pm 117)$ & $14( \pm 7)$ & $7( \pm 21)$ & $9( \pm 7)$ & DTM \\
\hline Mighei & $-12( \pm 108)$ & $107( \pm 204)$ & $24( \pm 11)$ & $-4( \pm 12)$ & $15( \pm 12)$ & Carlson et al. (2007) \\
\hline Murray & $129( \pm 108)$ & $109( \pm 204)$ & $11( \pm 11)$ & $-1( \pm 12)$ & $9( \pm 12)$ & Carlson et al. (2007) \\
\hline \multicolumn{7}{|l|}{$\mathrm{CV}_{\mathrm{ox}}$} \\
\hline Allende $_{\text {fused }}$ & $-67( \pm 92)$ & $-54( \pm 117)$ & $37( \pm 7)$ & $10( \pm 21)$ & $20( \pm 7)$ & DTM \\
\hline Allende $_{\text {acid }}$ & $-33( \pm 92)$ & $41( \pm 117)$ & $32( \pm 7)$ & $15( \pm 21)$ & $18( \pm 7)$ & DTM \\
\hline Grosnaja & $64( \pm 108)$ & $189( \pm 204)$ & $38( \pm 11)$ & $-3( \pm 12)$ & $15( \pm 12)$ & Carlson et al. (2007) \\
\hline Mokoia & $58( \pm 108)$ & $164( \pm 204)$ & $-14( \pm 11)$ & $-2( \pm 12)$ & $-4( \pm 12)$ & Carlson et al. (2007) \\
\hline Vigarano $_{\text {red }}$ & $10( \pm 92)$ & $-58( \pm 117)$ & $38( \pm 7)$ & $-11( \pm 21)$ & $19( \pm 7)$ & DTM \\
\hline \multicolumn{7}{|l|}{ CI } \\
\hline Orgueil 1 & - & $109( \pm 138)$ & $53( \pm 12)$ & $-19( \pm 29)$ & $23( \pm 11)$ & WWU \\
\hline Orgueil 2 & $-161( \pm 92)$ & $-44( \pm 117)$ & $22( \pm 7)$ & $6( \pm 21)$ & $16( \pm 7)$ & DTM \\
\hline Orgueil & $-39( \pm 108)$ & $168( \pm 204)$ & $31( \pm 11)$ & $8( \pm 12)$ & $15( \pm 12)$ & Carlson et al. (2007) \\
\hline
\end{tabular}


Fig. 1

2 The $\mathrm{Ba}$ isotopic composition of $\mathrm{SiC}$ grains from the $\mathrm{KJB}$ fraction (Murchison SiC-enriched sample), where $3 \delta \delta^{\mathrm{i}} \mathrm{Ba} /{ }^{136} \mathrm{Ba}=\left[\left({ }^{\mathrm{i}} \mathrm{Ba} /{ }^{136} \mathrm{Ba}\right)_{\text {measured }} /\left({ }^{\mathrm{B}} \mathrm{Ba} /{ }^{136} \mathrm{Ba}\right)_{\text {solar }}-1\right] \times 10^{3}$. Errors are $1 \sigma$ and are smaller than the symbols. Data is 4 from Ávila et al. (2013).

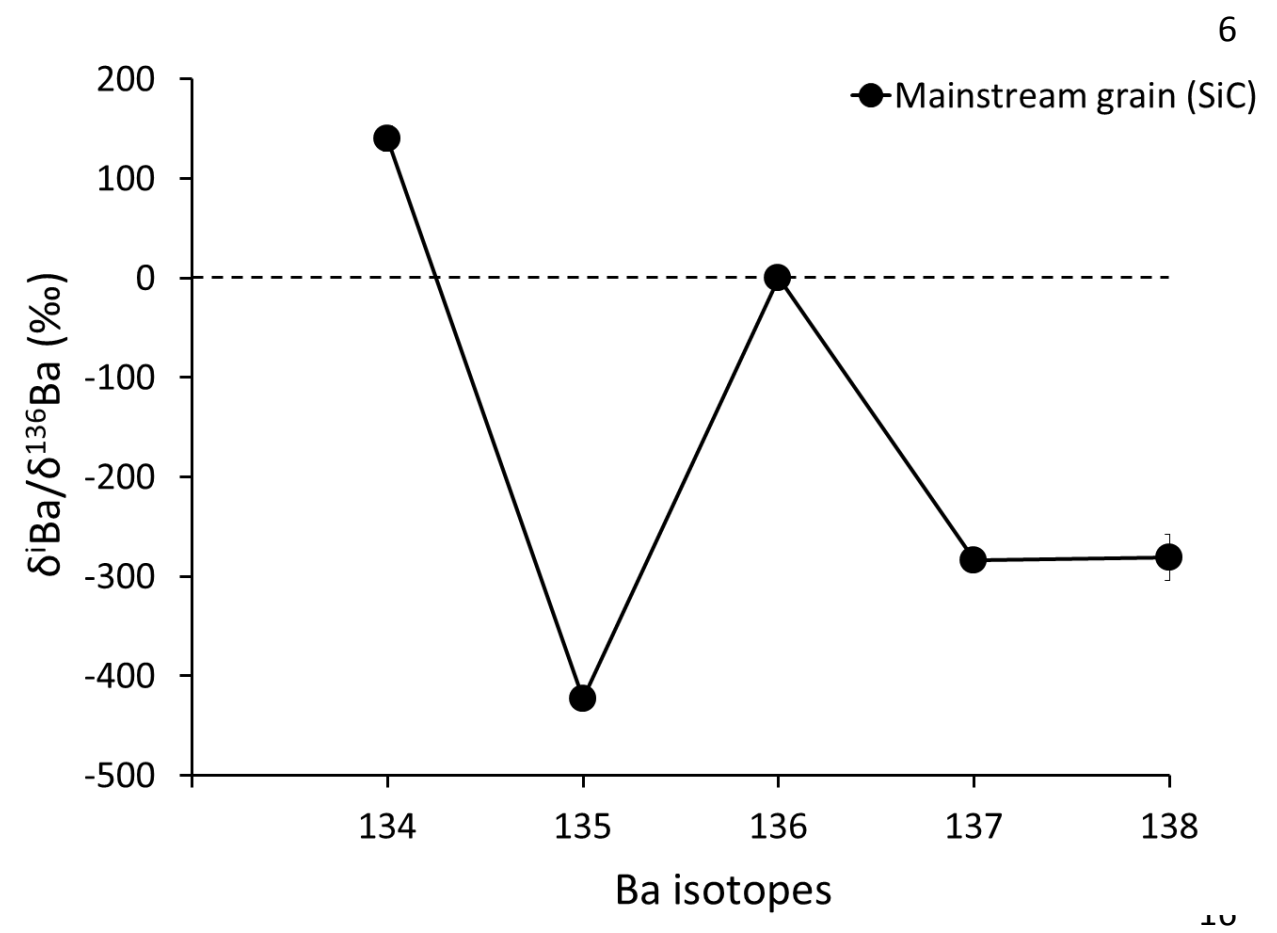

17

18 
Fig. 2

20 Back scatter electron images of (a) a $\sim 10 \mathrm{mg}$ sample of pure, synthetic $\mathrm{SiC}$ that was exposed to the laser for $\sim 3 \mathrm{~s}$.

21 Partial melting of the $\mathrm{SiC}$ is evident; (b) a $\sim 10 \mathrm{mg}$ aliquot of Lancé which was exposed to laser fusion for $\sim 15 \mathrm{~s}$ and

22 displays a quenched texture, indicating complete melting of the sample.

23

24
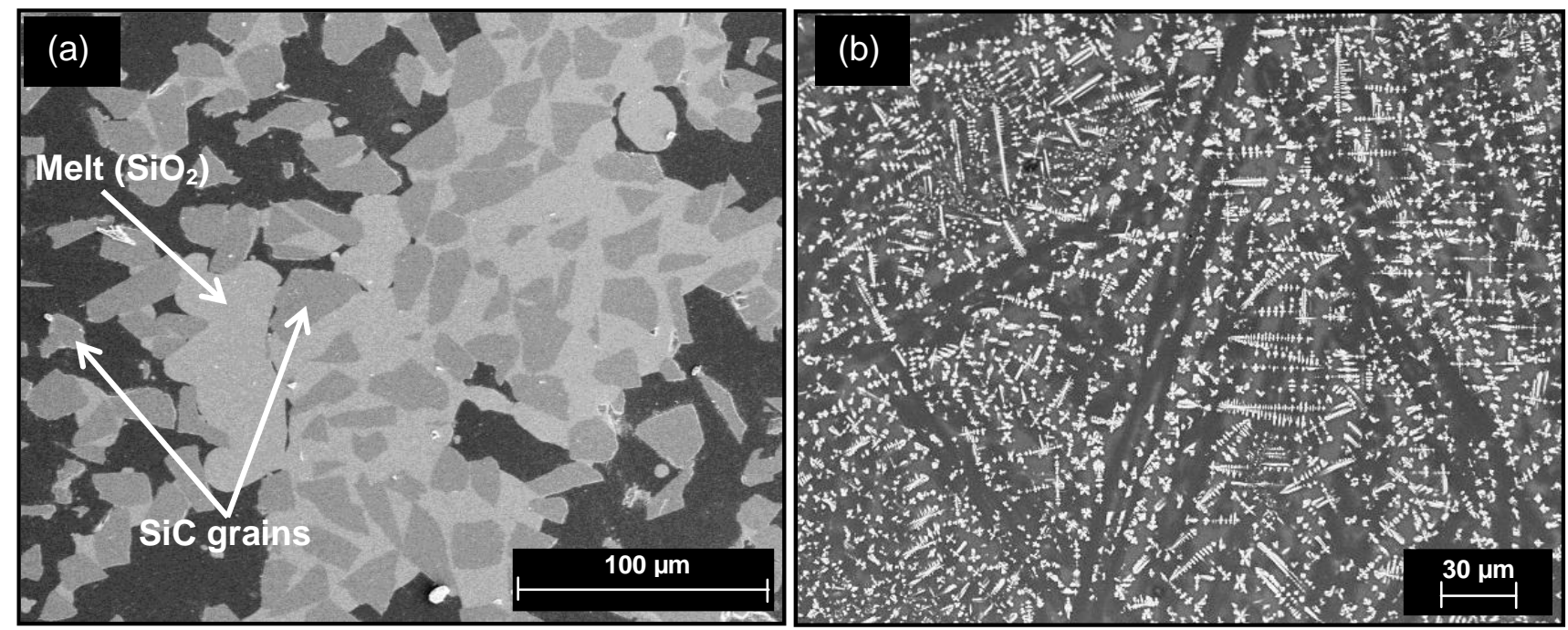

32

33

34 
Fig. 3

The average Ba isotope ratios (in ppm) of individual filament measurements of (a) terrestrial basalt (DU17) and (b) a selection of eucrites. Barium data are normalized to ${ }^{134} \mathrm{Ba} /{ }^{138} \mathrm{Ba}=0.03371$ (De Bièvre and Taylor, 1993; Andreasen and Sharma, 2007). The shaded area indicates the external precision as determined from the $2 \sigma$ of the population of replicate standard measurements. The error bars represent the $2 \sigma$ of the replicate sample analyses in ppm where multiple measurements of the same sample were possible. Where only one measurement of the sample was possible, the error bars represent $2 \sigma$ of the replicate standard analyses in ppm. The values and errors for ${ }^{132} \mathrm{Ba}$ data have been divided by 10 to plot on this scale.
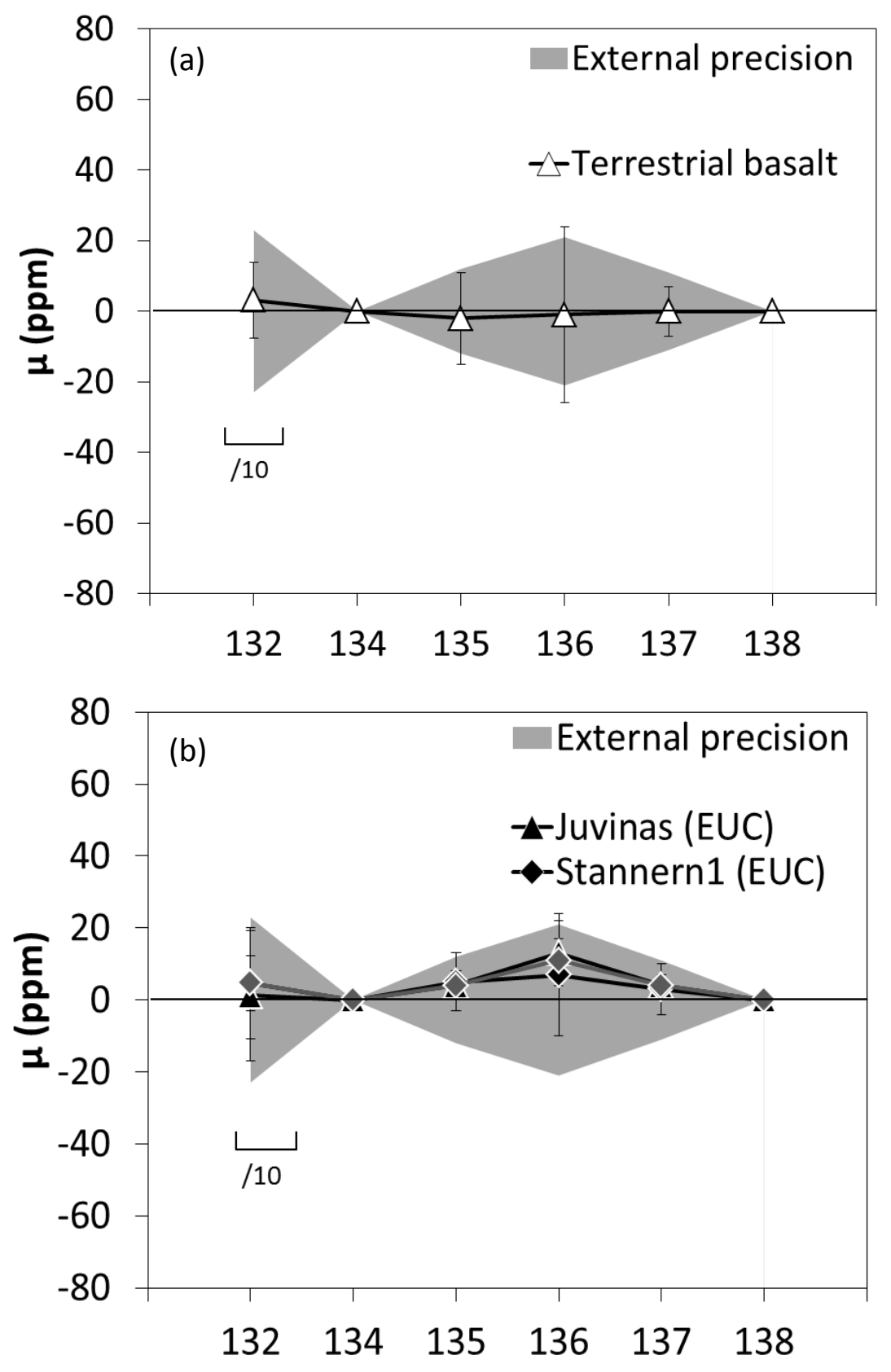
Fig. 4

The average $\mathrm{Ba}$ isotope ratios (in ppm) of individual filament measurements of (a) ordinary chondrites, (b) Martin meteorites, and (c-e) carbonaceous and enstatite chondrites. Barium data are normalized to ${ }^{134} \mathrm{Ba} /{ }^{138} \mathrm{Ba}=0.03371$ (De Bièvre and Taylor, 1993; Andreasen and Sharma, 2007). The shaded area indicates the external precision as determined from the $2 \sigma$ of the population of replicate standard measurements. The error bars represent the $2 \sigma$ of the replicate sample analyses in ppm where multiple measurements of the same sample were possible. Where only one measurement of the sample was possible, the error bars represent $2 \sigma$ of the replicate standard analyses in ppm. The values and errors for ${ }^{130} \mathrm{Ba}$ and ${ }^{132} \mathrm{Ba}$ data have been divided by 10 to plot on this scale. For (d) Allende_fused is the sample that underwent laser fusion prior to acid digestion. Allende_acid is the sample which did not undergo laser fusion prior to acid digestion.
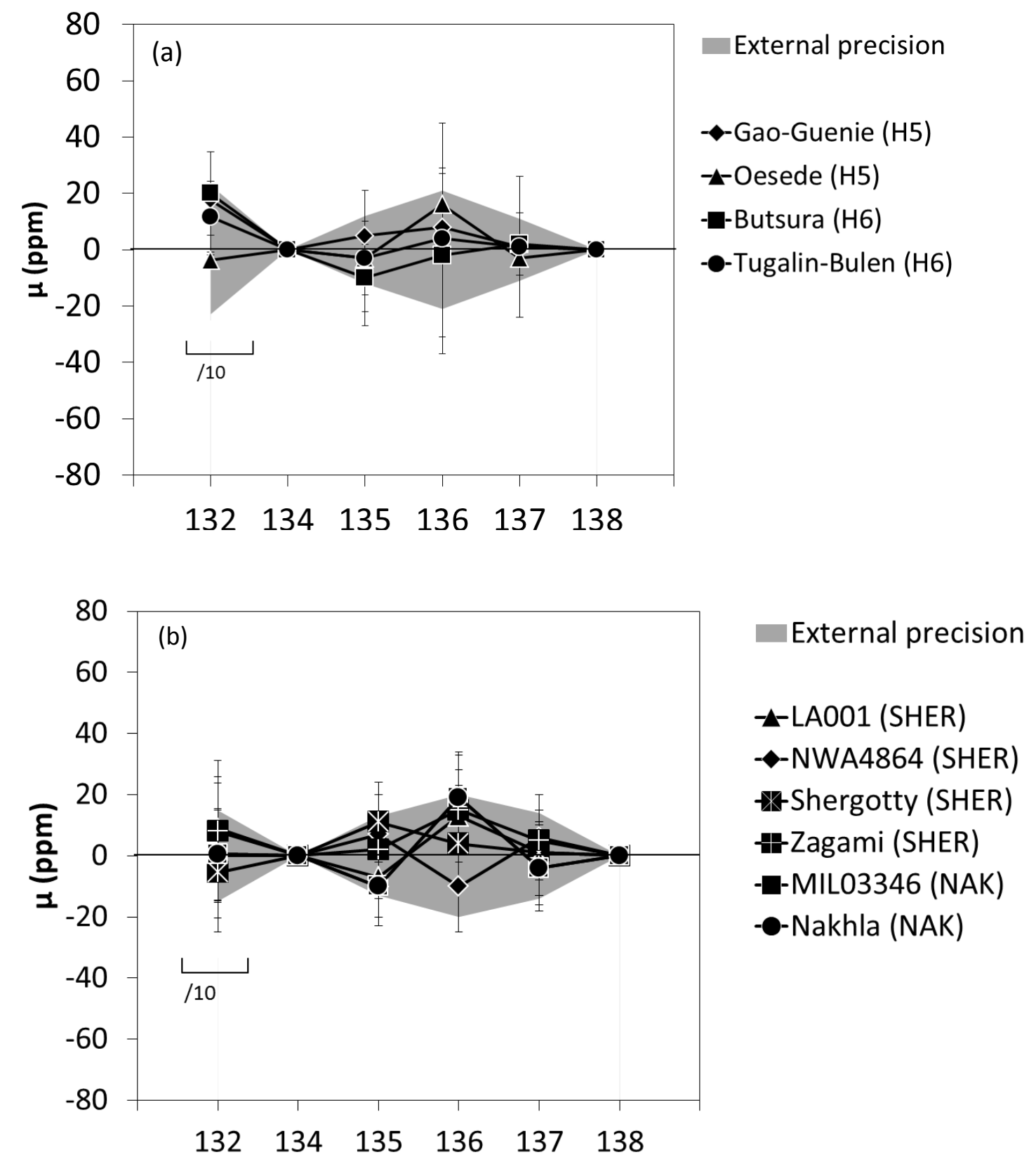


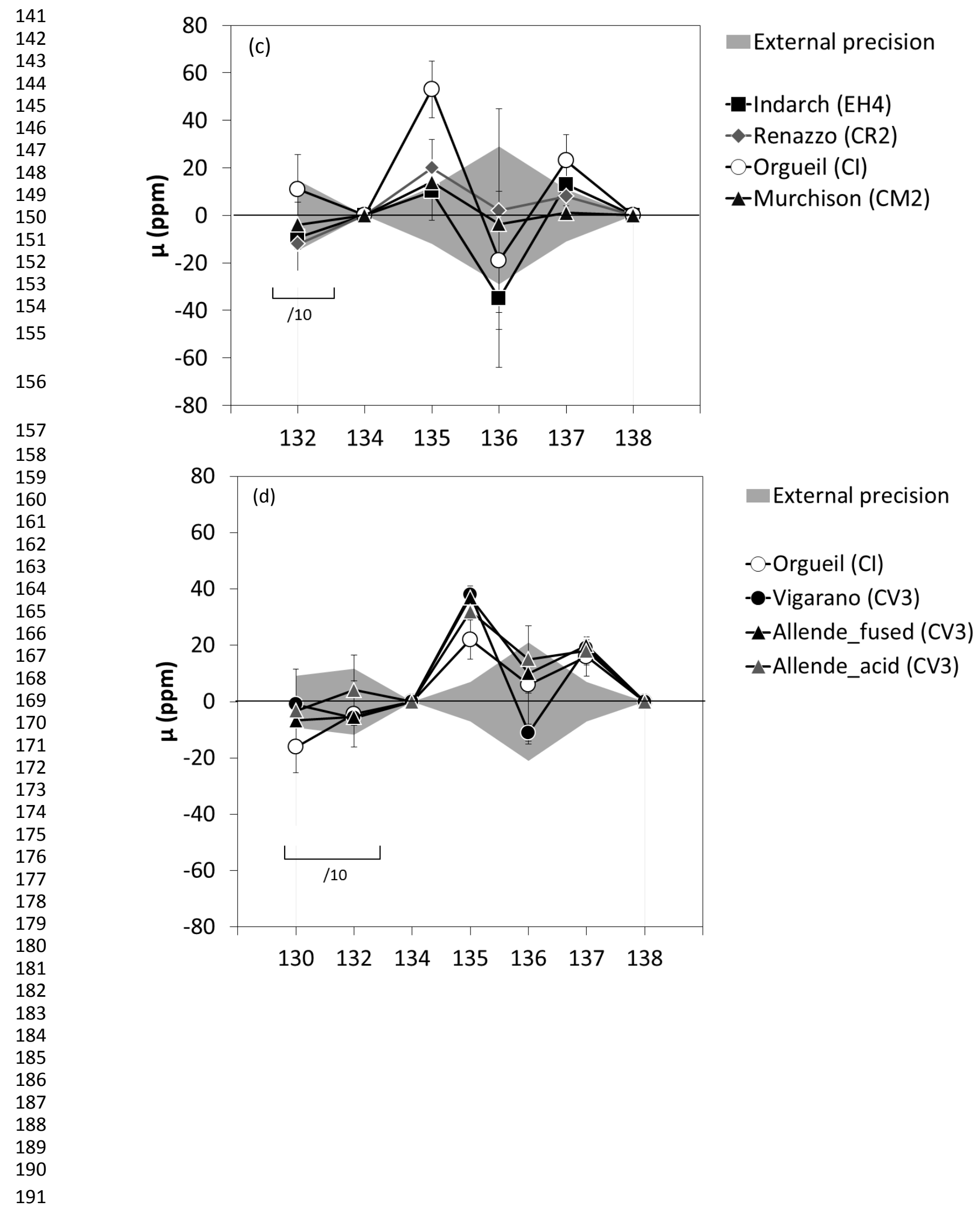




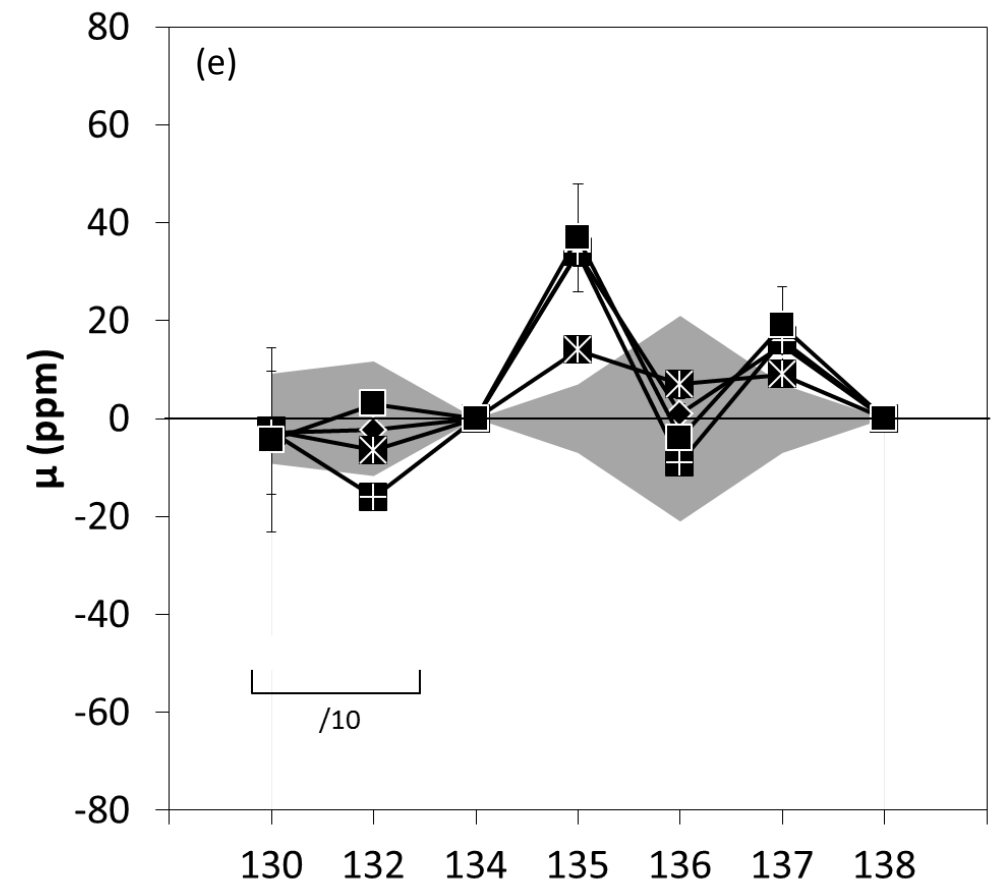

External precision

194

- Cold Bokkeveld (CM2)

*-Mighei (CM2)

\#-Kainsaz (CO3.2)

-mance (CO3.5) 